EUROPEAN ORGANIZATION FOR NUCLEAR RESEARCH

CERN-PH-EP/2004-026

June 14, 2004

\title{
Measurement of the Cross Section of W-boson pair production at LEP
}

The L3 Collaboration

\begin{abstract}
The cross section of $\mathrm{W}$-boson pair-production is measured with the L3 detector at LEP. In a data sample corresponding to a total luminosity of $629.4 \mathrm{pb}^{-1}$, collected at centre-of-mass energies ranging from 189 to $209 \mathrm{GeV}, 9834$ four-fermion events with $\mathrm{W}$ bosons decaying into hadrons or leptons are selected. The total cross section is measured with a precision of $1.4 \%$ and agrees with the Standard Model expectation. Assuming charged-lepton universality, the branching fraction for hadronic $\mathrm{W}$-boson decays is measured to be: $\mathrm{Br}(\mathrm{W} \rightarrow$ hadrons $)=67.50 \pm 0.42$ (stat.) \pm 0.30 (syst.) \%, in agreement with the Standard Model. Differential cross sections as a function of the $\mathrm{W}^{-}$production angle are also measured for the semi-leptonic channels qqe $\nu$ and qq $\mu \nu$.
\end{abstract}

Submitted to Phys. Lett. B 


\section{Introduction}

From 1996 until the year 2000, the centre-of-mass energy, $\sqrt{s}$, of the LEP $\mathrm{e}^{+} \mathrm{e}^{-}$collider at CERN was increased in several steps from $161 \mathrm{GeV}$ to $209 \mathrm{GeV}$. These energies, being above the kinematic threshold of $\mathrm{W}$-boson pair production, allow detailed studies of this process.

To lowest order within the Standard Model [1], three charged-current Feynman diagrams, shown in Figure 1 and referred to as CC03 [2-4], yield four-fermion final states via W-boson pair production: $t$-channel $\nu$ exchange and $s$-channel $\gamma$ and Z-boson exchange. W bosons decay into a quark-antiquark pair or a lepton-antilepton pair, denoted here as qq, $\ell \nu(\ell=\mathrm{e}, \mu, \tau)$ or, in general, $\mathrm{ff}$ for both $\mathrm{W}^{+}$and $\mathrm{W}^{-}$decays. This Letter describes measurements of all fourfermion final states $\ell \nu \ell \nu, q q \ell \nu$ and qqqq mediated by $\mathrm{W}$-boson pair production. The presence of additional photons in the final state is not excluded. Contributions to the production of fourfermion final states arising from other neutral- or charged-current Feynman diagrams are small. At the current level of statistical accuracy, interference terms are sizable for the 151 chargedand neutral-current diagrams contributing to the $\ell \nu \ell \nu$ final states, for the 20 charged-current diagrams contributing to the qqe $\nu$ final state and for the 214 charged- and neutral-current diagrams contributing to the qqqq final state [2-4].

It is conventional to quote results for the CC03 subset of diagrams, including the effect of initial-state radiation. As four-fermion states produced by all diagrams are measured, a suitable reweighting technique, described in the following, is applied to extract these results.

W-boson decay branching fractions and the total W-boson pair-production cross section are determined with improved precision as compared to earlier L3 measurements at $\sqrt{s}=$ $161-189 \mathrm{GeV}$ [5-8]. Comparable results were reported by other LEP experiments [9].

The differential cross sections for the qqe $\nu$ and qq $\mu \nu$ final states, as a function of the $\mathrm{W}^{-}$ production angle with respect to the direction of the incoming electron, are also derived.

\section{Data and Monte Carlo Samples}

The results presented in this Letter are based on the full luminosity collected by the L3 detector [10] during the high-energy runs of the LEP collider. The measurement of the total luminosity, $\mathcal{L}$, follows the procedure described in Reference 11.

The data collected at $\sqrt{s}=192-209 \mathrm{GeV}$ are analysed in seven $\sqrt{s}$ bins, as detailed in Table 1. The corresponding centre-of-mass energies are known with a precision of about $50 \mathrm{MeV}$ [12]. Results based on data collected at $\sqrt{s}=189 \mathrm{GeV}$ were already published [8] but are reanalysed here since improved Monte Carlo programs are now available for signal simulation: KandY [13] and RacoonWW [14]. The KandY generator combines the four-fermion generator KORALW [15] with the $\mathcal{O}(\alpha)$ corrections to W-boson pair production as implemented in the YFSWW3 [16] program. These corrections are calculated using the leading-pole approximation [17]. The RacoonWW Monte Carlo program implements such corrections in the double-pole approximation with similar accuracy and it is used for the estimation of systematic uncertainties.

All KandY Monte Carlo samples used in this analysis are generated using the full set of Feynman diagrams contributing to a specific four-fermion final state. The KandY program provides the matrix elements on an event-by-event basis for different contributions including, for example, the CC03 subset of diagrams or the $\mathcal{O}(\alpha)$ corrections. This feature is extensively used in the following, both to derive quantities at CC03 level and for the assessment of systematic uncertainties. For example, the CC03-level efficiencies are calculated by reweighting every 
event with the factor $w_{\mathrm{CC} 03}=|\mathcal{M}(\mathrm{CC} 03)|^{2} /|\mathcal{M}(4 \mathrm{f})|^{2}$, where $\mathcal{M}(\mathrm{CC} 03)$ and $\mathcal{M}(4 \mathrm{f})$ are the matrix elements of the CC03 subset of diagrams and of the full set, respectively. The same events, reweighted by the factor $1-w_{\mathrm{CC} 03}$, describe the remaining four-fermion contribution not arising from W-boson pair production. In the following they are considered as background. As a cross-check, selection efficiencies are also derived using the EXCALIBUR [18] four-fermion generator.

The following Monte Carlo event generators are used to simulate the background processes: KK2f [19], PYTHIA [20], BHAGENE3 [21] and BHWIDE [22] for fermion-pair production, denoted as $\mathrm{e}^{+} \mathrm{e}^{-} \rightarrow \mathrm{f} \overline{\mathrm{f}}(\gamma)$; TEEGG [23] for radiative $\mathrm{e}^{+} \mathrm{e}^{-} \rightarrow \mathrm{e}^{+} \mathrm{e}^{-} \gamma(\gamma)$ events; DIAG36 [24] and LEP4F [25] for two-photon collisions with lepton-pair final states and PHOJET [26] for two-photon collisions with hadronic final states.

Quark fragmentation and hadronisation processes are simulated using PYTHIA. Its parameters are tuned to describe hadronic $\mathrm{Z}$ decays at $\sqrt{s}=91 \mathrm{GeV}[27]$. A dedicated parameter set, derived from a light-quark Z-decay data sample, is used for the W-boson pair-production simulations. Bose-Einstein correlations between hadrons from $\mathrm{W}$ decays are simulated using the LUBOEI $\mathrm{BE}_{32}$ model [28], with Bose-Einstein correlations only between hadrons originating from the same $\mathrm{W}$ boson, as supported by our study [29].

The response of the L3 detector is modelled with the GEANT [30] detector simulation program which includes the effects of energy loss, multiple scattering and showering in the detector material. Hadronic showers are simulated with the GHEISHA [31] program. Time-dependent detector inefficiencies, as monitored during data taking, are included in the simulations.

\section{Four-Fermion Event Selection}

The selections of four-fermion final states are designed to mimimise the uncertainty on the cross section of each channel. They are chosen to be mutually exclusive, by using complementary cuts, in order to avoid double counting of events.

Electrons are identified as energy depositions in the BGO electromagnetic calorimeter having an electromagnetic shower shape and matching in azimuth a track reconstructed in the central tracking chamber. Muons are identified as tracks reconstructed in the muon chambers, which

point back to the interaction vertex. Tracks which match a minimum-ionising-particle signature in the calorimeters are also retained as muon candidates and denoted as MIPs. Jets arising from hadronic tau decays are reconstructed using a jet-clustering algorithm in a cone of $15^{\circ}$ halfopening angle [32]. The momentum of the neutrino in qq $\ell \nu$ events is identified with the missing momentum vector of the event. Hadronic jets corresponding to quarks are reconstructed using the Durham jet algorithm [33]. In the $\mathrm{e}^{+} \mathrm{e}^{-} \rightarrow \mathrm{qq} \ell \nu$ selections, the hadronic jets are formed from energy depositions and tracks not belonging to the reconstructed lepton.

Efficiencies are evaluated for each $\sqrt{s}$ point in the form of 10 by 10 matrices relating events at CC03 level to those at reconstruction level. An example is given in Table 2 for $\sqrt{s}=206.5 \mathrm{GeV}$. Selection efficiencies at other centre-of-mass energies are only marginally different.

The number of selected events and background contributions are detailed in Table 3. A more detailed description of all selections is given below. 


\subsection{The $\ell \nu \ell \nu$ Selection}

The event selection for the process $\mathrm{e}^{+} \mathrm{e}^{-} \rightarrow \ell \nu \ell \nu$ requires two charged leptons and missing energy due to the neutrinos. The selection depends on whether the event contains zero, one or two identified electrons or muons, referred to as jet-jet, lepton-jet and lepton-lepton classes. For the lepton-jet and jet-jet classes, only the most energetic jets are retained as tau candidates. Electrons, muons and jets from hadronic tau decays are identified within the polar angular range $|\cos \theta|<0.96$, where $\theta$ is the lepton angle with respect to the beam direction. For events with one or two electrons, one electron is required to satisfy $|\cos \theta|<0.92$ in order to reduce the background from Bhabha scattering. For the jet-jet class, the two most energetic jets must also satisfy $|\cos \theta|<0.92$.

The acoplanarity, defined as the complement of the angle between the directions of the two leptons in the plane transverse to the beam direction, must be greater than 8 degrees for the lepton-lepton and lepton-jet classes and 14 degrees for the jet-jet class. These criteria suppress the dominating backgrounds from lepton-pair production and cosmic rays. The leptons must have a signal in the scintillator time-of-flight counters compatible with the beam crossing. The total momentum transverse to the beam direction, $P_{\mathrm{t}}$, must be greater than $8 \mathrm{GeV}$.

Events belonging to the lepton-lepton class are selected by requiring an energy of at least $25 \mathrm{GeV}$ for the more energetic lepton and $5 \mathrm{GeV}$ for the less energetic one. For the lepton-jet class, the energies of the lepton and of the jet must exceed $20 \mathrm{GeV}$ and $8 \mathrm{GeV}$, respectively. For the jet-jet class, the energies of the most energetic and second most energetic jets must be greater than $20 \mathrm{GeV}$ and $6 \mathrm{GeV}$, respectively.

The selected sample has a purity of $72 \%$ at $\sqrt{s}=206.5 \mathrm{GeV}$. The remaining background is dominated by lepton production in two-photon collisions (50\%) and lepton-pair production $(24 \%)$. The distributions of the acoplanarity and of the missing momentum transverse to the beam direction for the lepton-lepton class are shown in Figure 2.

\subsection{The qqe $\nu$ Selection}

The event selection for the process $\mathrm{e}^{+} \mathrm{e}^{-} \rightarrow$ qqe $\nu$ requires an identified electron of at least $20 \mathrm{GeV}$, high particle-multiplicity and large missing momentum.

The missing momentum direction must point well inside the detector with a polar angle, $\theta_{\text {miss }}$, such that $\left|\cos \theta_{\text {miss }}\right|<0.95$. The reconstructed jet-jet and lepton-neutrino masses, referred to as $M_{\mathrm{jj}}$ and $M_{\mathrm{e} \nu}$, must be greater than $45 \mathrm{GeV}$ and $63 \mathrm{GeV}$, respectively. The latter cut is used to discriminate between $\mathrm{e}^{+} \mathrm{e}^{-} \rightarrow \mathrm{qqe} \nu$ and $\mathrm{e}^{+} \mathrm{e}^{-} \rightarrow \mathrm{qq} \tau \nu$ events with $\tau \rightarrow$ e $\nu \nu$. To further suppress the dominant background from the $\mathrm{e}^{+} \mathrm{e}^{-} \rightarrow \mathrm{q} \overline{\mathrm{q}}(\gamma)$ process, which is planar, the directions of the electron and of the two jets are required to subtend a solid angle of less than $5.3 \mathrm{sr}$.

The purity of the selection is $98 \%$ at $\sqrt{s}=206.5 \mathrm{GeV}$. The accepted background not originating from $\mathrm{W}$-boson pair production is dominated by $\mathrm{e}^{+} \mathrm{e}^{-} \rightarrow \mathrm{qqe} \nu$ final states $(71 \%)$ and

$\mathrm{e}^{+} \mathrm{e}^{-} \rightarrow \mathrm{q} \overline{\mathrm{q}}(\gamma)$ events (29\%). The distributions of the energy of the electron and of $\left|\cos \left(\theta_{\text {miss }}\right)\right|$ are shown in Figure 2.

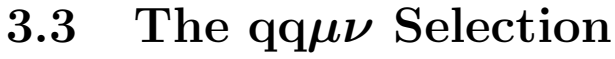

The event selection for the process $\mathrm{e}^{+} \mathrm{e}^{-} \rightarrow \mathrm{qq} \mu \nu$ requires high particle-multiplicity, an identified muon or a MIP, and large missing momentum. 
The jet-jet mass must satisfy $25 \mathrm{GeV}<M_{\mathrm{jj}}<125 \mathrm{GeV}$ for events with identified muons and $50 \mathrm{GeV}<M_{\mathrm{jj}}<98 \mathrm{GeV}$ for events with MIPs. The muon-neutrino reconstructed mass, $M_{\mu \nu}$, is used as a discriminant against $\mathrm{e}^{+} \mathrm{e}^{-} \rightarrow \mathrm{qq} \tau \nu$ events with $\tau \rightarrow \mu \nu_{\tau} \nu_{\mu}$. Its value is required to exceed $53 \mathrm{GeV}$. This cut is not applied for events containing MIPs.

The discrimination against $\mathrm{e}^{+} \mathrm{e}^{-} \rightarrow \mathrm{qq} \tau \nu$ events is further enhanced by requiring the variable $P^{\star}=\left|p_{\mu}\right|-10 \mathrm{GeV}\left(\cos \theta^{\star}+1\right)$, where $p_{\mu}$ is the momentum of the muon and $\theta^{\star}$ is the decay angle of the muon in the reconstructed $\mathrm{W}$-boson rest frame, to satisfy $P^{\star}>18.5 \mathrm{GeV}$. This requirement is loosened to $P^{\star}>15 \mathrm{GeV}$ for events with MIPs.

The $\mathrm{e}^{+} \mathrm{e}^{-} \rightarrow \mathrm{q} \overline{\mathrm{q}}(\gamma)$ process is a potentially large source of background. It is reduced by exploiting the fact that it originates muons close to the jets and that the total missing momentum, if any, points towards the beam direction. The product of $\psi_{\mu j}$, the angle between the muon and the closest jet, and $\sin \theta_{\text {miss }}$ is required to be greater than 5.5 degrees for events with muons and greater than 20 degrees for events with MIPs.

Background due to $\mathrm{q} \overline{\mathrm{q}} \mu^{+} \mu^{-}$final states from Z-boson pair production in events containing MIPs is rejected by requiring the relativistic velocity of the reconstructed $W$ bosons to be greater than a $\sqrt{s}$-dependent value, ranging from 0.34 to 0.49 .

The purity of the selection is $98 \%$ at $\sqrt{s}=206.5 \mathrm{GeV}$. The residual background, not originating from $\mathrm{W}$-boson pair production, is dominated by Z-boson pair-production events $(52 \%)$ and $\mathrm{e}^{+} \mathrm{e}^{-} \rightarrow \mathrm{q} \overline{\mathrm{q}}(\gamma)$ events $(31 \%)$. The distributions of $M_{\mathrm{jj}}$ and of $\psi_{\mu j} \times \sin \theta_{\text {miss }}$ are shown in Figure 3.

\subsection{The qq $\tau \nu$ Selection}

The event selection for the process $\mathrm{e}^{+} \mathrm{e}^{-} \rightarrow \mathrm{qq} \tau \nu$ is based on the identification of an isolated low-momentum electron, muon, or narrow jet in a hadronic environment with large missing energy.

Events are selected requiring $P_{\mathrm{t}}>10 \mathrm{GeV}, 30 \mathrm{GeV}<M_{\mathrm{jj}}<110 \mathrm{GeV}$ and the mass recoiling against the two-jet system to be greater than $35 \mathrm{GeV}$.

Events are classified according to the presence of isolated electrons or muons with an energy of more than $5 \mathrm{GeV}$. MIPs are not considered as tau candidates.

For leptonically-decaying tau candidates, cuts on $M_{\mathrm{e} \nu}$ and $M_{\mu \nu}$, complementary to those described in Sections 3.2 and 3.3, are applied. These cuts are chosen so as to minimise correlations in the measured W-boson branching fractions.

If no electrons or muons are found, a search for a tau-jet is performed using a neural network which exploits the distinctive characteristics of a hadronic tau decay: low multiplicity, small jet opening angle, low jet mass and high electromagnetic fraction of the jet energy. The jet with the highest neural-network output is retained as the tau-lepton candidate. For these events, additional requirements are applied in order to reduce the dominant background from $\mathrm{e}^{+} \mathrm{e}^{-} \rightarrow$ $\mathrm{q} \overline{\mathrm{q}}(\gamma)$ events. If $P_{\mathrm{t}}<20 \mathrm{GeV}$, the neural-network output of the tau-jet candidate is required to be near to that expected for a tau-jet. At most three charged tracks are allowed to form the tau-jet candidate. The polar angle of the missing momentum must satisfy $\left|\cos \theta_{\text {miss }}\right|<0.91$. The solid angle subtended by the directions of the tau-jet candidate and the other two jets must be less than 6 sr.

Among the events selected at $\sqrt{s}=206.5 \mathrm{GeV}, 62 \%$ come from $\mathrm{e}^{+} \mathrm{e}^{-} \rightarrow \mathrm{qq} \tau \nu$ W-boson pair-production processes and $21 \%$ from other final states of the $\mathrm{W}$-boson pair production. The background is dominated by $\mathrm{e}^{+} \mathrm{e}^{-} \rightarrow \mathrm{q} \overline{\mathrm{q}}(\gamma)$ events (54\%) and $\mathrm{e}^{+} \mathrm{e}^{-} \rightarrow \mathrm{qqe} \nu$ final states not originating from W-boson pair production (46\%). The distributions of $M_{\ell \nu}$ and $M_{\mathrm{jj}}$ are shown 
in Figure 3.

\subsection{The qqqq Selection}

The event selection for the process $\mathrm{e}^{+} \mathrm{e}^{-} \rightarrow$ qqqq requires hadronic events with little missing energy, high multiplicity and a four-jet topology.

The Durham jet-resolution parameter $y_{34}$, for which the event topology changes from three to four jets, is required to be greater than 0.0015. The events are clustered into four jets and a kinematic fit, assuming four-momentum conservation, is used to improve energy and angle resolutions.

A neural network is trained to discriminate against the dominant $\mathrm{e}^{+} \mathrm{e}^{-} \rightarrow \mathrm{q} \overline{\mathrm{q}}(\gamma)$ background. Ten variables are used in the neural network: the spherocity [34], the lowest jet-multiplicity, $y_{34}$, the energies of the most and of the least energetic jets, the difference between the energies of the second and the third most energetic jets, the broadenings [35] of the most and of the least energetic jets, the probability of the kinematic fit and the sum of the cosines of the six angles between the four jets. The dominant background is due to $\mathrm{e}^{+} \mathrm{e}^{-} \rightarrow \mathrm{q} \overline{\mathrm{q}}(\gamma)$ events with four reconstructed jets, mainly coming from $\mathrm{e}^{+} \mathrm{e}^{-} \rightarrow \mathrm{q} \overline{\mathrm{q} g g}$ events. We find that the four-jet rate in $\mathrm{e}^{+} \mathrm{e}^{-} \rightarrow \mathrm{q} \overline{\mathrm{q}}(\gamma)$ events is not well described by our MC simulations, and a comparison with data is used to determine this background. Data and Monte Carlo distributions of the $y_{34}$ variable in hadronic $\mathrm{Z}$ decays collected at $\sqrt{s}=91 \mathrm{GeV}$ are compared and their ratio is used to reweight the $\mathrm{e}^{+} \mathrm{e}^{-} \rightarrow \mathrm{q} \overline{\mathrm{q}}(\gamma)$ Monte Carlo events at higher energies throughout the rest of the analysis. The resulting accepted number of $\mathrm{e}^{+} \mathrm{e}^{-} \rightarrow \mathrm{q} \overline{\mathrm{q}}(\gamma)$ events, for a neural network output greater than 0.6 , is increased by $12.7 \%$.

Requiring the neural-network output to be greater than 0.6 yields a sample purity of $80 \%$ at $\sqrt{s}=206.5 \mathrm{GeV}$ with a background dominated by the $\mathrm{e}^{+} \mathrm{e}^{-} \rightarrow \mathrm{q} \overline{\mathrm{q}}(\gamma)(59 \%)$ and Z-boson pair-production (41\%) processes. The distributions of some of the neural-network inputs and of its output, peaking at one for the signal and at zero for the background, are shown in Figure 4.

\section{Fit Method}

The CC03-level cross sections, $\sigma_{j}$, of the signal processes $j$ are determined simultaneously in a single maximum-likelihood fit, taking cross-feed between different final states into account.

For the purely leptonic final states, the fit procedure determines six different cross sections corresponding to all possible lepton-flavour combinations. Since the statistics for the $\ell \nu \ell \nu$ final state is low, the sum of these six cross sections is quoted in the following as the cross section for the process $\mathrm{e}^{+} \mathrm{e}^{-} \rightarrow \ell \nu \ell \nu$.

The total likelihood is given by the product of Poissonian probabilities, $P\left(N_{i}, \mu_{i}\right)$, to observe $N_{i}$ events in the $i$-th final state, as listed in Table 3. The expected number of events for selection $i, \mu_{i}$, is calculated as:

$$
\mu_{i}=\left(\sum_{j=1}^{10} \epsilon_{i j} \sigma_{j}+\sum_{k=1}^{N_{i}^{\mathrm{bg}}} \epsilon_{i k}^{\mathrm{bg}} \sigma_{k}^{\mathrm{bg}}\right) \mathcal{L},
$$

where $\epsilon_{i j}$ is the CC03-level efficiency of selection $i$ to accept events from process $j, \sigma_{k}^{\mathrm{bg}}$ is the cross section of the $k$-th background process, selected with efficiency $\epsilon_{i k}^{\mathrm{bg}}$. The $N_{i}^{\mathrm{bg}}$ background processes for selection $i$ also include four-fermion final states not originating from W-boson pair production. 
For the $\mathrm{e}^{+} \mathrm{e}^{-} \rightarrow$ qqqq process, the Poissonian probability is replaced by the likelihood as a function of the signal cross section derived from a fit to the neural-network output distribution. In this fit the $\mathrm{e}^{+} \mathrm{e}^{-} \rightarrow \mathrm{q} \overline{\mathrm{q}}(\gamma)$ background contribution is fixed to the value derived directly from

data by performing a fit with both the signal and background normalisations left free. The results for the $\mathrm{e}^{+} \mathrm{e}^{-} \rightarrow \mathrm{q} \overline{\mathrm{q}}(\gamma)$ background cross sections are shown in Table 4 . These values are in good agreement with the Monte Carlo predictions. As a cross-check, the $\mathrm{e}^{+} \mathrm{e}^{-} \rightarrow$ qqqq cross section is also determined by repeating the full fit after applying a cut on the output of the neural network at 0.6 , which minimises the expected statistical uncertainty. All values agree well with those derived from the neural-network fit.

\section{$5 \quad$ Systematic Uncertainties}

In addition to the uncertainty on the luminosity measurement [11] and that due to limited Monte Carlo statistics, which affect all final states in common, the remaining sources of systematic effects in the measurement of W-boson pair-production cross sections are divided into two classes: uncertainties in the detector response and theoretical uncertainties. The latter come mainly from the knowledge and modelling of the hadronisation processes. A summary of the systematic uncertainties from all considered sources is given in Table 5 for $\sqrt{s}=206.5 \mathrm{GeV}$. Values at different $\sqrt{s}$ are only marginally different. Details about the assessment of the systematic uncertainties are discussed below.

A possible source of systematic uncertainty arises from the accuracy of the Monte Carlo modelling of the detector response. For the semi-leptonic and fully-leptonic final states, this uncertainty is evaluated by varying the positions of the selection cuts for each channel. The variation of the cut positions is chosen so as to span several times the resolution of the studied variable. Each variable is considered in turn and the corresponding change in the measured cross sections are evaluated. For variables which are correlated, for instance visible energies and transverse momenta, the largest variation is retained. For the selected variables, the expected statistical uncertainty on the newly-selected data sample is subtracted from the observed variation and the sum in quadrature of all results is retained as systematic uncertainty. Most of the systematic effects are related to the resolution of the missing momentum. In addition, the electron/photon discrimination represents also a sizable source of systematic uncertainty for the qqe $\nu$ final state.

For the qqqq selection, the systematic uncertainty on the neural-network output is estimated by re-evaluating the input variables of the neural network after smearing and scaling the measurements of energy depositions and tracks in the simulation according to the uncertainties on their resolutions.

The relative systematic uncertainty on the measured cross section, assigned to detector response and modelling, varies from $1.0 \%$ to $2.0 \%$ depending on the final state.

As a cross-check, changes in efficiency due to variations of the detector calibration within its uncertainty, are also studied. The calibration is studied using samples of di-lepton and di-jet events, collected during the calibration runs at $\sqrt{s}=91 \mathrm{GeV}$ and at higher energies. The results of this study show a much smaller effect than the cut-variation technique. The trigger inefficiency, as well as its uncertainty, is found to be negligible in all channels.

Fragmentation and hadronisation uncertainties may affect both the signal efficiency and the $\mathrm{e}^{+} \mathrm{e}^{-} \rightarrow \mathrm{q} \overline{\mathrm{q}}(\gamma)$ background estimation. The modelling of the signal hadronisation is studied comparing the selection efficiencies obtained with different hadronisation models: PYTHIA, HERWIG [36] and ARIADNE [37]. The average difference with respect to PYTHIA gives a 
systematic uncertainty on the measured cross section of $0.5 \%$ to $1.2 \%$, dependent on the final state.

The effect of the hadronisation uncertainty in $\mathrm{e}^{+} \mathrm{e}^{-} \rightarrow \mathrm{q} \overline{\mathrm{q}}(\gamma)$ background events is also studied by comparing PYTHIA, HERWIG and ARIADNE. It is found to be negligible for qq $\ell \nu$ final states. In the qqqq final state, the hadronisation uncertainty affects mainly the four-jet rate as described in Section 3.5. Half of the effect due to the $y_{34}$ reweighting is assigned as systematic uncertainty. It corresponds to $0.9 \%$ of the measured $\mathrm{e}^{+} \mathrm{e}^{-} \rightarrow$ qqqq cross section.

Other sources of theoretical uncertainties in the qqqq channel arise from correlations among final-state hadrons such as Bose-Einstein correlations and colour reconnection. The modelling of Bose-Einstein correlations between hadrons from W-boson decays may affect the selection efficiencies. In previous studies [29] we have measured the strength of Bose-Einstein correlations between hadrons originating from the same $\mathrm{W}$ boson in semi-leptonic $\mathrm{W}$ decays. Its value is significantly different from zero and in good agreement with that for light-quark Z decays and also with that of the LUBOEI BE 32 model [28] used in our Monte Carlo simulations. The systematic uncertainty derived from the uncertainty of this strength is found to be negligible. Bose-Einstein correlations between particles originating from different $\mathrm{W}$ bosons are strongly disfavoured in $\mathrm{e}^{+} \mathrm{e}^{-} \rightarrow$ qqqq events [29]. Their measured strength is restricted to at most a quarter of the strength expected in the $\mathrm{BE}_{32}$ model with full correlations. Allowing correlations with such a strength yields negligible changes in the measured cross sections.

Extreme models of colour reconnection between the hadronic systems in qqqq events are disfavoured by data $[38,39]$. The influence of colour reconnection is estimated using the models implemented in HERWIG [40], ARIADNE [41] (model 1 and model 2) and PYTHIA (model SK I with reconnection parameter $k=0.6$ [42]). The ARIADNE-2 model is compared to a modified version of the ARIADNE-1 model, so that in both models the shower cascade is performed in two phases with an identical energy cut-off parameter. The average difference of $0.19 \%$ with respect to PYTHIA is assigned as systematic uncertainty on the measured $\mathrm{e}^{+} \mathrm{e}^{-} \rightarrow$ qqqq cross section.

The theoretical uncertainties on the cross sections of the background processes, namely hadron production in two-photon collisions (50\%), neutral-current four-fermion processes $(5 \%)$ and fermion-pair production (1\%) lead to systematic uncertainties of $0.1 \%$ to $0.4 \%$. In the determination of the $\mathrm{e}^{+} \mathrm{e}^{-} \rightarrow$ qqqq cross sections, the $\mathrm{e}^{+} \mathrm{e}^{-} \rightarrow \mathrm{q} \overline{\mathrm{q}}(\gamma)$ background levels are directly measured from data and the corresponding uncertainties, as reported in Table 4, are propagated to the final results.

The dependence of the selection efficiencies on the mass and width of the $\mathrm{W}$ boson, $m_{\mathrm{W}}$ and $\Gamma_{\mathrm{W}}$, is studied using Monte Carlo samples simulated with different $m_{\mathrm{W}}$ and $\Gamma_{\mathrm{W}}$ values. The propagation of the world-average uncertainties on these two parameters, $40 \mathrm{MeV}$ on $m_{\mathrm{W}}$ and $60 \mathrm{MeV}$ on $\Gamma_{\mathrm{W}}$ [43], is taken as systematic uncertainty. It corresponds to a less than $0.3 \%$ effect.

The systematic uncertainty on initial-state radiation (ISR), due to its approximate leading$\log \mathcal{O}\left(\alpha^{3}\right)$ treatment in KandY, is investigated by re-evaluating the signal efficiencies for Monte Carlo events reweighted by $\left|\mathcal{M}\left[\mathcal{O}\left(\alpha^{2}\right)\right]\right|^{2} /\left|\mathcal{M}\left[\mathcal{O}\left(\alpha^{3}\right)\right]\right|^{2}$. The effect is found to be negligible. As a cross-check, the Monte Carlo events are also reweighted by $10 \%$ in the presence of ISR photons with energies or transverse momenta exceeding $100 \mathrm{MeV}$. In both cases, the effect is negligible.

Final-state radiation (FSR) is implemented in KandY using the PHOTOS package [44] based on the leading-log approximation. The PHOTOS package is inaccurate in the hard noncollinear region. The related systematic uncertainty is estimated by determining the selection 
efficiencies using Monte Carlo events whose weights are reduced by $50 \%$ in the presence of FSR photons with energy greater than $30 \mathrm{GeV}$. An effect between $0.1 \%$ and $0.2 \%$ is observed and retained as systematic uncertainty.

Uncertainties due to the implementation of virtual $\mathcal{O}(\alpha)$ corrections in the KandY program are tested comparing signal efficiencies to those obtained with the RacoonWW program. No sizable effect is observed.

Correlations among all sources of systematic uncertainties are taken into account in the following results.

\section{Results}

\subsection{Single-Channel Cross Sections}

Fits are performed to derive ten cross sections, one for each final state. No assumption is made concerning the W-boson branching fractions. The results, summing up all fully leptonic final states and including statistical and systematic uncertainties, are listed in Table 3. The Standard Model agrees well with these results. Since the efficiency matrix of Table 2 contains non-zero offdiagonal elements, the measured cross sections are correlated. The largest correlations, $-10.3 \%$ and $-17.6 \%$, are between the $\mathrm{e}^{+} \mathrm{e}^{-} \rightarrow \mathrm{qq} \tau \nu$ and $\mathrm{e}^{+} \mathrm{e}^{-} \rightarrow \mathrm{qqe} \nu$ and between the $\mathrm{e}^{+} \mathrm{e}^{-} \rightarrow \mathrm{qq} \tau \nu$ and $\mathrm{e}^{+} \mathrm{e}^{-} \rightarrow \mathrm{qq} \mu \nu$ cross sections, respectively. All other correlations are less than $1 \%$.

\subsection{Total Cross Section and Branching Fractions}

For the determination of the CC03 cross section of W-boson pair production, $\sigma_{\mathrm{Ww}}$, the signal cross sections $\sigma_{j}$ are replaced by the product $r_{j} \sigma_{\mathrm{WW}}$. The ratios $r_{j}$ are given in terms of the $\mathrm{W}$ boson decay branching fractions, $\operatorname{Br}(\mathrm{W} \rightarrow \mathrm{qq})$ and $\operatorname{Br}(\mathrm{W} \rightarrow \ell \nu)$, as follows: $r_{\mathrm{qqqq}}=[\operatorname{Br}(\mathrm{W} \rightarrow$

$\mathrm{qq})]^{2}, r_{\mathrm{qq} \ell \nu}=2 \mathrm{Br}(\mathrm{W} \rightarrow \mathrm{qq}) \operatorname{Br}(\mathrm{W} \rightarrow \ell \nu)$, and $r_{\ell \nu \ell \nu}=[\operatorname{Br}(\mathrm{W} \rightarrow \ell \nu)]^{2}$ for same-flavour leptons or $2 \mathrm{Br}(\mathrm{W} \rightarrow \ell \nu) \mathrm{Br}\left(\mathrm{W} \rightarrow \ell^{\prime} \nu\right)$ otherwise.

Results for the cross sections of the reactions $\mathrm{e}^{+} \mathrm{e}^{-} \rightarrow \ell \nu \ell \nu, \mathrm{e}^{+} \mathrm{e}^{-} \rightarrow \mathrm{qq} \ell \nu$ and $\mathrm{e}^{+} \mathrm{e}^{-} \rightarrow \mathrm{qqqq}$, assuming charged-lepton universality, are obtained as shown in Table 6 . The total cross sections, $\sigma_{\mathrm{WW}}$, are then derived assuming the Standard Model W-boson decay branching fractions [3] and are also reported in Table 6 together with the Standard Model expectations. Our previous measurements at $\sqrt{s}$ of $161 \mathrm{GeV}[5], 172 \mathrm{GeV}$ [6], $183 \mathrm{GeV}$ [7] and these results are compared in Figure 5 to the Standard Model expectation as calculated with the Monte Carlo programs YFSWW3 and RacoonWW. The two predictions agree with our data and are consistent within a theoretical uncertainty of $0.5 \%[45]$ for $\sqrt{s} \geq 170 \mathrm{GeV}$.

The ratios of the measured cross sections to the Standard Model predictions of the YFSWW3 program are also shown in Figure 5. Their combined value, $R$, is:

$$
R=0.992 \pm 0.011 \pm 0.009 \pm 0.005
$$

where the first uncertainty is statistical, the second systematic and the third theoretical.

For the determination of $\mathrm{W}$-boson decay branching fractions, the data collected at lower centre-of-mass energies are also included. The sum of the hadronic and the three leptonic branching fractions is constrained to unity. The branching fractions are first determined without the assumption of charged-lepton universality, with the results listed in Table 7 . The hypothesis of charged-lepton universality is tested and the probability of getting a $\chi^{2}$ greater than that 
observed is $0.8 \%$ differing by 2.6 standard deviations from this hypothesis. Assuming chargedlepton universality, the hadronic $\mathrm{W}$-boson decay branching fraction is:

$$
\operatorname{Br}(\mathrm{W} \rightarrow \mathrm{qq})=67.50 \pm 0.42 \pm 0.30 \%
$$

where the first uncertainty is statistical and the second systematic. The W-boson decay branching fractions depend on the six elements $V_{i j}$ of the Cabibbo-Kobayashi-Maskawa matrix $V$ [46] not involving the top quark [3]:

$$
1 / \mathrm{Br}(\mathrm{W} \rightarrow \ell \nu)=3+3\left[1+\alpha_{\mathrm{s}}\left(m_{\mathrm{W}}\right) / \pi\right] \sum_{i=\mathrm{u}, \mathrm{c} ; j=\mathrm{d}, \mathrm{s}, \mathrm{b}}\left|V_{i j}\right|^{2}
$$

where $\alpha_{\mathrm{s}}$ is the strong coupling constant. Using $\alpha_{\mathrm{s}}=0.119 \pm 0.002$ [43], our measurements correspond to:

$$
\sum_{i=\mathrm{u}, \mathrm{c} ; j=\mathrm{d}, \mathrm{s}, \mathrm{b}}\left|V_{i j}\right|^{2}=2.002 \pm 0.038 \pm 0.027
$$

where the first uncertainty is statistical and the second systematic.

Using the current world-average values and uncertainties of the other matrix elements, not imposing the unitarity of the $V$ matrix, $\left|V_{\mathrm{cs}}\right|$ is derived as:

$$
\left|V_{\mathrm{cs}}\right|=0.977 \pm 0.020 \pm 0.014
$$

where the first uncertainty is statistical and the second systematic. The systematic uncertainty includes the uncertainties on $\alpha_{\mathrm{s}}$ and on the other matrix elements [43].

\subsection{Differential Cross Section}

The combined differential cross section for the $\mathrm{e}^{+} \mathrm{e}^{-} \rightarrow \mathrm{qqe} \nu$ and $\mathrm{e}^{+} \mathrm{e}^{-} \rightarrow \mathrm{qq} \mu \nu$ channels, as a function of $\cos \theta_{\mathrm{W}^{-}}$, where $\theta_{\mathrm{W}^{-}}$is the $\mathrm{W}^{-}$production angle with respect to the direction of the incoming electrons, is measured for different $\sqrt{s}$ from $183 \mathrm{GeV}$ to $209 \mathrm{GeV}$. These two channels are used because the lepton charge tags the W-boson charge with high purity.

Four energy bins are considered:

$$
\begin{aligned}
& 180.0-184.0 \mathrm{GeV}, 184.0-194.0 \mathrm{GeV} \\
& 194.0-204.0 \mathrm{GeV}, 204.0-209.0 \mathrm{GeV} \text {. }
\end{aligned}
$$

These are chosen so as to minimise the difference between the average slope of the differential cross section in each bin and the slope corresponding to the luminosity-weighted average centreof-mass energies: $\langle\sqrt{s}\rangle=182.7,189.0,198.3$ and $205.9 \mathrm{GeV}$, respectively. In each energy range, ten $\cos \theta_{\mathrm{W}^{-}}$bins are studied. The variable $\cos \theta_{\mathrm{W}^{-}}$is reconstructed from the measurements of the jet and lepton angles and energies [47]. Monte Carlo events are then used to extract the differential cross section. Ambiguities might arise in the presence of additional photons in the generated events, and the $\cos \theta_{\mathrm{W}^{-}}$angle is then defined following the $\gamma$-recombination scheme [45]:

- all photons inside a cone of 5 degrees half-opening angle with respect to the beam direction are treated as invisible;

- the combined mass of each photon with electrons, muons and quarks is calculated. If the smallest combined mass is less than $5 \mathrm{GeV}$ or the energy of the photon is less than $1 \mathrm{GeV}$, the momentum of the photon is added to that of the fermion and the photon is discarded. 
The measured cross sections are corrected to CC03-level with the additional restriction of $20^{\circ}<\theta_{\ell^{ \pm}}<160^{\circ}$, where $\theta_{\ell^{ \pm}}$is the angle between the charged lepton and the beam direction.

The observed $\cos \theta_{\mathrm{W}^{-}}$distributions are corrected to generator level, after background subtraction, by using bin-by-bin correction factors and the cross sections in each $\cos \theta_{\mathrm{W}^{-}}$bin are determined as listed in Table 8 and plotted in Figure 6.

As a cross-check, a full matrix unfolding from reconstruction to generator level is also used. Since the migration matrix is almost diagonal, with bin-to-bin migration effects at the level of $20 \%$ at most, the results are in perfect agreement with the simple bin-by-bin correction method.

The potential bias of implicitly assuming the Standard Model $\cos \theta_{\mathrm{W}^{-}}$distribution in the correction factors, is studied using simulated samples with modified $\cos \theta_{\mathrm{W}^{-}}$behaviour and found to be negligible. Another bias could arise directly from the W-boson pair-production Monte Carlo generator used to estimate the correction factors. No difference between KORALW and YFSWW3 programs is observed, hence no additional systematic uncertainty is assigned.

Charge-confusion effects, which affect the reconstruction of the W-boson direction, are taken into account. The residual uncertainty, obtained by comparing data and Monte Carlo expectations on Z-peak samples [47], is retained as a systematic uncertainty in addition to those affecting the total W-boson pair-production cross section.

The systematic uncertainty is taken to be fully correlated between $\cos \theta_{\mathrm{W}^{-}}$bins and energy points.

\section{Conclusions}

In a data sample corresponding to an integrated luminosity of $629.4 \mathrm{pb}^{-1}$, collected at centreof-mass energies ranging from $189 \mathrm{GeV}$ to $209 \mathrm{GeV}$, W-boson pair-production cross sections are measured by selecting four-fermion events and found to be in agreement with Standard Model expectations.

The branching fractions for leptonic W decays are measured for each lepton generation. Assuming charged-lepton universality, the branching fraction for hadronic $\mathrm{W}$ decays is measured to be: $67.50 \pm 0.42$ (stat.) \pm 0.30 (syst.) \% . Combining all $\sqrt{s}$ points, the ratio $R$ of the measured total W-boson pair-production cross section with respect to the theoretical prediction is found to be $R=0.992 \pm 0.011$ (stat.) \pm 0.009 (syst.) \pm 0.005 (theo.).

Differential cross sections as a function of the $\mathrm{W}^{-}$production angle are also measured and found to be in good agreement with Standard Model predictions. 


\section{References}

[1] S. L. Glashow, Nucl. Phys. 22 (1961) 579;

S. Weinberg, Phys. Rev. Lett. 19 (1967) 1264;

A. Salam, in Elementary Particle Theory, ed. N. Svartholm, Stockholm, Almquist and Wiksell (1968), 367.

[2] D. Bardin et al., Nucl. Phys. (Proc. Suppl.) B 37 (1994) 148;

F.A. Berends et al., Nucl. Phys. (Proc. Suppl.) B 37 (1994) 163.

[3] W. Beenakker et al., in Physics at LEP 2, Report CERN 96-01 (1996), eds G. Altarelli, T. Sjöstrand, F. Zwirner, Vol. 1, p. 79.

[4] D. Bardin et al., in Physics at LEP 2, Report CERN 96-01 (1996), eds G. Altarelli, T. Sjöstrand, F. Zwirner, Vol. 2, p. 3.

[5] L3 Collab., M. Acciarri et al., Phys. Lett. B 398 (1997) 223.

[6] L3 Collab., M. Acciarri et al., Phys. Lett. B 407 (1997) 419.

[7] L3 Collab., M. Acciarri et al., Phys. Lett. B 436 (1998) 437.

[8] L3 Collab., M. Acciarri et al., Phys. Lett. B 496 (2000) 19.

[9] ALEPH Collab., A. Heister et al., preprint CERN-PH-EP/2004-012 (2004), (submitted to Eur. Phys. Jour. C);

DELPHI Collab., J. Abdallah et al., preprint CERN-EP/2003-071 (2003), (submitted to Eur. Phys. Jour. C);

OPAL Collab., K. Ackerstaff et al., Phys. Lett. B 389 (1996) 416;

OPAL Collab., K. Ackerstaff et al., Eur. Phys. Jour. C 1 (1998) 425;

OPAL Collab., G. Abbiendi et al., Eur. Phys. Jour. C 8 (1999) 191;

OPAL Collab., G. Abbiendi et al., Phys. Lett. B 493 (2000) 249.

[10] L3 Collab., B. Adeva et al., Nucl. Instr. Meth. A 289 (1990) 35;

L3 Collab., O. Adriani et al., Physics Reports 236 (1993) 31;

M. Chemarin et al., Nucl. Instr. Meth. A 349 (1994) 345;

M. Acciarri et al., Nucl. Instr. Meth. A 351 (1994) 300;

G. Basti et al., Nucl. Instr. Meth. A 374 (1996) 293;

A. Adam et al., Nucl. Instr. Meth. A 383 (1996) 342;.

[11] I.C. Brock et al., Nucl. Instr. Meth. A 381 (1996) 236.

[12] The LEP Energy Working Group, Calibration of centre-of-mass energies at LEP 2 for a precise measurement of the $W$ boson mass, in preparation;

http://lepecal.web.cern.ch/LEPECAL.

[13] KandY runs concurrently KORALW version 1.51 and YFSWW3 version 1.16.

S. Jadach et al., Comp. Phys. Comm. 140 (2001) 475.

[14] RacoonWW version 1.3 is used.

A. Denner et al., Nucl. Phys. B 587 (2000) 67;

A. Denner et al., preprint hep-ph/0006307 (2000). 
[15] KORALW version 1.51 is used.

S. Jadach et al., Comp. Phys. Comm. 119 (1999) 272.

[16] YFSWW3 version 1.16 is used.

Jadach et al., Phys. Rev. D65 (2002) 093010.

[17] A. Aeppli et al., Nucl. Phys. B 428 (1994) 126.

[18] EXCALIBUR version 1.11 is used.

R. Kleiss and R. Pittau, Comp. Phys. Comm. 83 (1994) 141.

[19] KK2f version 4.14 and 4.19 are used.

S. Jadach et al., Comp. Phys. Comm. 130 (2000) 260;

S. Jadach et al., Phys. Rev. D 63 (2001) 113009.

[20] PYTHIA versions 5.722 and 6.1 are used.

T. Sjöstrand, preprint CERN-TH/7112/93 (1993), revised 1995;

T. Sjöstrand, Comp. Phys. Comm. 82 (1994) 74;

T. Sjöstrand et al., Comp. Phys. Comm. 135 (2001) 238.

[21] BHAGENE version 3.0 is used.

J.H. Field, Phys. Lett. B 323 (1994) 432;

J.H. Field and T. Riemann, Comp. Phys. Comm. 94 (1996) 53.

[22] BHWIDE version 1.01 is used.

S. Jadach et al., Phys. Rev. D 40 (1989) 3582,

S. Jadach et al., Comp. Phys. Comm. 70 (1992) 305,

S. Jadach et al., Phys. Lett. B 390 (1997) 298.

[23] TEEGG version 7.1 is used.

D. Karlen, Nucl. Phys. B 289 (1987) 23.

[24] DIAG36 version 1.0 is used.

F. A. Berends et al., Nucl. Phys. B 253 (1985) 441.

[25] LEP4F version 2.0 is used.

J.A.M. Vermaseren et al., Phys. Rev. D 19 (1979) 137;

J.A.M. Vermaseren, Nucl. Phys. B 229 (1983) 347.

[26] PHOJET version 1.05 is used.

R. Engel, Z. Phys. C 66 (1995) 203;

R. Engel and J. Ranft, Phys. Rev. D 54 (1996) 4244.

[27] L3 Collab., P. Achard et al., Studies of Hadronic Event Structure in $\mathrm{e}^{+} \mathrm{e}^{-}$Annihilations from $30 \mathrm{GeV}$ to $209 \mathrm{GeV}$ with the L3 Detector, preprint CERN-PH-EP-2004-024, hepex/0406049 (2004) to appear in Physics Reports.

[28] L. Lönnblad and T. Sjöstrand, Eur. Phys. J. C 2 (1998) 165.

[29] L3 Collab., P. Achard et al., Phys. Lett. B 547 (2002) 139.

[30] GEANT Version 3.15 is used.

R. Brun et al., preprint CERN-DD/EE/84-1 (1984), revised 1987. 
[31] H. Fesefeldt, RWTH Aachen Preprint PITHA 85/02 (1985).

[32] H.J. Daum et al., Z. Phys. C 8 (1981) 167.

[33] S. Catani et al., Phys. Lett. B 269 (1991) 432;

S. Bethke et al., Nucl. Phys. B 370 (1992) 310.

[34] H. Georgi and M. Machacek, Phys. Rev. Lett. 39 (1977) 1237.

[35] P.E.L. Rakow and B.R. Webber, Nucl. Phys. B 191 (1981) 63.

[36] HERWIG version 6.202 is used.

G. Marchesini et al., Comp. Phys. Comm. 67 (1992) 465;

G. Corcella et al., JHEP 101 (2001) 010.

[37] ARIADNE version 4.12 is used.

L. Lönnblad, Comp. Phys. Comm. 71 (1992) 15.

[38] L3 Collab., P. Achard et al., Phys. Lett. B 561 (2003) 202.

[39] L3 Collab., P. Achard et al., Phys. Lett. B 581 (2004) 19.

[40] B. Webber, J. Phys. G 24 (1998) 287.

[41] L. Lönnblad, Z. Phys. C 70 (1996) 107.

[42] T. Sjöstrand and V.A. Khoze, Phys. Rev. Lett. 72 (1994) 28,

T. Sjöstrand and V.A. Khoze, Z. Phys. C 62 (1994) 281,

T. Sjöstrand and V.A. Khoze, Eur. Phys. J. C 6 (1999) 271.

[43] K. Hagiwara et al., Phys. Rev. D 66 (2002) 1.

[44] PHOTOS version 2.3 is used.

E. Barberio and Z. Wạs, Comp. Phys. Comm. 79 (1994) 291.

[45] M. W. Grünewald et al., preprint hep-ph/0005309 (2000).

[46] N. Cabibbo, Phys. Rev. Lett. 10 (1963) 531;

M. Kobayashi and T. Maskawa, Prog. Theor. Phys. 49 (1973) 652.

[47] L3 Collab., P. Achard et al., Phys. Lett. B 586 (2004) 151. 


\section{The L3 Collaboration:}

P.Achard ${ }^{20}$ O.Adriani, ${ }^{17}$ M.Aguilar-Benitez ${ }^{25}$ J.Alcaraz ${ }^{25}$ G.Alemanni, ${ }^{23}$ J.Allaby ${ }^{18}$ A.Aloisio, ${ }^{29}$ M.G.Alviggi ${ }^{29}$ H.Anderhub, ${ }^{49}$ V.P.Andreev, ${ }^{6,34}$ F.Anselmo, A.Arefiev, ${ }^{28}$ T.Azemoon, T.Aziz, P.Bagnaia, ${ }^{39}$ A.Bajo, ${ }^{25}$ G.Baksay, ${ }^{26}$ L.Baksay ${ }^{26}$ S.V.Baldew, ${ }^{2}$ S.Banerjee, ${ }^{9}$ Sw.Banerjee, ${ }^{4}$ A.Barczyk ${ }^{49,47}$ R.Barillère, ${ }^{18}$ P.Bartalini ${ }^{23}$ M.Basile, N.Batalova, ${ }^{46}$ R.Battiston, ${ }^{33}$ A.Bay, ${ }^{23}$ F.Becattini, ${ }^{17}$ U.Becker ${ }^{13}$ F.Behner, ${ }^{49}$ L.Bellucci ${ }^{17}$ R.Berbeco, ${ }^{3}$ J.Berdugo, ${ }^{25}$ P.Berges, ${ }^{13}$ B.Bertucci, ${ }^{33}$ B.L.Betev, ${ }^{49}$ M.Biasini, ${ }^{33}$ M.Biglietti, ${ }^{29}$ A.Biland, ${ }^{49}$ J.J.Blaising, ${ }^{4}$ S.C.Blyth, ${ }^{35}$ G.J.Bobbink, ${ }^{2}$ A.Böhm, L.Boldizsar, ${ }^{12}$ B.Borgia, ${ }^{19}$ S.Bottai, ${ }^{17}$ D.Bourilkov, ${ }^{49}$ M.Bourquin, ${ }^{20}$ S.Braccini ${ }^{20}$ J.G.Branson, ${ }^{41}$ F.Brochu, J.D.Burger, ${ }^{13}$ W.J.Burger, ${ }^{33}$ A.Button, X.D.Cai ${ }^{3}{ }^{3}$ M.Capell, ${ }^{13}$ G.Cara Romeo, G.Carlino, ${ }^{29}$ A.Cartacci ${ }^{17}$ J.Casaus, ${ }^{25}$ F.Cavallari ${ }^{39}{ }^{2}$ N.Cavallo, ${ }^{36}$ C.Cecchi ${ }^{33}$ M.Cerrada, ${ }^{25}$ M.Chamizo, ${ }^{20}$ Y.H.Chang, ${ }^{44}$ M.Chemarin, ${ }^{24}$ A.Chen, ${ }^{44}$ G.Chen, G.M.Chen, H.F.Chen, ${ }^{22}$ H.S.Chen, G.Chiefari ${ }^{29}$ L.Cifarelli, ${ }^{40}$ F.Cindolo, ${ }^{8}$ I.Clare, ${ }^{13}$ R.Clare ${ }^{38}$ G.Coignet, ${ }^{4}$ N.Colino, ${ }^{25}$ S.Costantini, ${ }^{39}$ B.de la Cruz ${ }^{25}$ S.Cucciarelli, ${ }^{33}$ J.A.van Dalen, ${ }^{31}$ R.de Asmundis, ${ }^{29}$ P.Déglon, ${ }^{20}$ J.Debreczeni, ${ }^{12}$ A.Degré, ${ }^{4}$ K.Dehmelt ${ }^{26}$ K.Deiters, ${ }^{47}$ D.della Volpe, ${ }^{29}$ E.Delmeire ${ }^{20}$ P.Denes ${ }^{37}$ F.DeNotaristefani, ${ }^{39}$ A.De Salvo, ${ }^{49}$ M.Diemoz ${ }^{39}$ M.Dierckxsens, ${ }^{2}$ D.van Dierendonck, C.Dionisi ${ }^{39}$ M.Dittmar, ${ }^{49}$ A.Doria ${ }^{29}$ M.T.Dova, ${ }^{10, \#}$ D.Duchesneau, ${ }^{4}$ M.Duda, ${ }^{1}$ B.Echenard, ${ }^{20}$ A.Eline, ${ }^{18}$ A.El Hage, H.El Mamouni, ${ }^{24}$ A.Engler ${ }^{35}$ F.J.Eppling, ${ }^{13}$ P.Extermann, ${ }^{20}$ M.A.Falagan, ${ }^{25}$ S.Falciano, ${ }^{39}$ A.Favara, ${ }^{32}$ J.Fay, ${ }^{24}$ O.Fedin ${ }^{34}$ M.Felcini ${ }^{49}$ T.Ferguson, ${ }^{35}$ H.Fesefeldt, ${ }^{1}$ E.Fiandrini, ${ }^{33}$ J.H.Field, ${ }^{20}$ F.Filthaut, ${ }^{31}$ P.H.Fisher ${ }^{13}$ W.Fisher, ${ }^{37}$ I.Fisk, ${ }^{41}$ G.Forconi ${ }^{13}$ K.Freudenreich, ${ }^{49}$ C.Furetta, ${ }^{27}$ Yu.Galaktionov, ${ }^{28,13}$ S.N.Ganguli, P.Garcia-Abia, ${ }^{25}$ M.Gataullin, ${ }^{32}$ S.Gentile, ${ }^{39}$ S.Giagu ${ }^{39}$ Z.F.Gong, ${ }^{22}$ G.Grenier, ${ }^{24}$ O.Grimm, ${ }^{49}$ M.W.Gruenewald, ${ }^{16}$ M.Guida, ${ }^{40}$ V.K.Gupta, ${ }^{37}$ A.Gurtu, L.J.Gutay, ${ }^{46}$ D.Haas, D.Hatzifotiadou, T.Hebbeker, ${ }^{1}$ A.Hervé ${ }^{8}{ }^{8}$ J.Hirschfelder, ${ }^{35}$ H.Hofer, ${ }^{49}$ M.Hohlmann, ${ }^{26}$ G.Holzner, ${ }^{49}$ S.R.Hou, ${ }^{44}$ Y.Hu, ${ }^{31}$ B.N.Jin, L.W.Jones, P.de Jong, ${ }^{2}$ I.Josa-Mutuberría, ${ }^{25}$ M.Kaur, ${ }^{14}$ M.N.Kienzle-Focacci ${ }^{20}$ J.K.Kim ${ }^{43}$ J.Kirkby, ${ }^{18}$ W.Kittel ${ }^{31}$ A.Klimentov, ${ }^{13,28}$ A.C.König, ${ }^{31}$ M.Kopal, ${ }^{46}$ V.Koutsenko, ${ }^{13,28}$ M.Kräber, ${ }^{49}$ R.W.Kraemer, ${ }^{35}$ A.Krüger, ${ }^{48}$ A.Kunin ${ }^{13}{ }^{\text {P.Ladron de Guevara, }}{ }^{25}$ I.Laktineh $^{24}$ G.Landi ${ }^{17}$ M.Lebeau, ${ }^{18}$ A.Lebedev ${ }^{13}$ P.Lebrun, ${ }^{24}$ P.Lecomte, ${ }^{49}$ P.Lecoq ${ }^{18}$ P.Le Coultre ${ }^{49}$ J.M.Le Goff, ${ }^{18}$ R.Leiste ${ }^{48}$ M.Levtchenko, ${ }^{27}$ P.Levtchenko, ${ }^{34}$ C.Li ${ }^{22}$ S.Likhoded ${ }^{48}$ C.H.Lin,${ }^{44}$ W.T.Lin ${ }^{44}$ F.L.Linde ${ }^{2}$ L.Lista, ${ }^{29}$ Z.A.Liu, W.Lohmann, ${ }^{48}$ E.Longo, ${ }^{39}$ Y.S.Lu, ${ }^{7}$ C.Luci, ${ }^{39}$ L.Luminari, ${ }^{39}$ W.Lustermann, ${ }^{49}$ W.G.Ma ${ }^{22}$ L.Malgeri ${ }^{18}$ A.Malinin ${ }^{28}$ C.Maña, ${ }^{25}$ J.Mans, ${ }^{37}$ J.P.Martin, ${ }^{24}$ F.Marzano, ${ }^{39}$ K.Mazumdar, ${ }^{9}$ R.R.McNeil, S.Mele, ${ }^{18,29}$ L.Merola, ${ }^{29}$ M.Meschini, ${ }^{17}$ W.J.Metzger ${ }^{31}$ A.Mihul, ${ }^{11}$ H.Milcent ${ }^{18}$ G.Mirabelli, ${ }^{39}$ J.Mnich, ${ }^{1}$ G.B.Mohanty ${ }^{9}$ T.Moulik, ${ }^{9}$ G.S.Muanza, ${ }^{24}$ A.J.M.Muijs, ${ }^{2}$ B.Musicar ${ }^{41}$ M.Musy ${ }^{39}{ }^{\text {S.Nagy }}{ }^{15}$ R.Nandakumar, ${ }^{9}$ S.Natale ${ }^{20}$ M.Napolitano, ${ }^{29}$ F.Nessi-Tedaldi ${ }^{49}$ H.Newman, ${ }^{32}$ A.Nisati, ${ }^{39}$ T.Novak ${ }^{31}$ H.Nowak, ${ }^{48}$ R.Ofierzynski, ${ }^{49}$ G.Organtini, ${ }^{39}$ I.Pal, ${ }^{46}$ C.Palomares ${ }^{25}$ P.Paolucci ${ }^{29}$ R.Paramatti ${ }^{39}$ G.Passaleva ${ }^{17}$ S.Patricelli ${ }^{29}$ T.Paul ${ }^{10}$ M.Pauluzzi ${ }^{33}$ C.Paus ${ }^{13}$ F.Pauss,${ }^{49}$ M.Pedace ${ }^{39}$ S.Pensotti ${ }^{27}$ D.Perret-Gallix, B.Petersen, ${ }^{31}$ D.Piccolo, ${ }^{29}$ F.Pierella, ${ }^{8}$ M.Pioppi ${ }^{33}$ P.A.Piroué ${ }^{37}$ E.Pistolesi ${ }^{27}$ V.Plyaskin,${ }^{28}$ M.Pohl ${ }^{20}$ V.Pojidaev, ${ }^{17}$ J.Pothier, ${ }^{18}$ D.Prokofiev, ${ }^{34}$ J.Quartieri, ${ }^{40}$ G.Rahal-Callot ${ }^{49}$ M.A.Rahaman, P.Raics $^{15}$ N.Raja, R.Ramelli, ${ }^{49}$ P.G.Rancoita, ${ }^{27}$ R.Ranieri, ${ }^{17}$ A.Raspereza, ${ }^{48}$ P.Razis ${ }^{30}$ D.Ren, ${ }^{49}$ M.Rescigno, ${ }^{39}$ S.Reucroft ${ }^{10}$ S.Riemann, ${ }^{48}$ K.Riles, B.P.Roe, L.Romero, ${ }^{25}$ A.Rosca, ${ }^{48}$ C.Rosemann, ${ }^{1}$ C.Rosenbleck, S.Rosier-Lees, S.Roth, J.A.Rubio, ${ }^{18}$ G.Ruggiero, ${ }^{17}$ H.Rykaczewski, ${ }^{9}$ A.Sakharov ${ }^{49}$ S.Saremi, ${ }^{6}$ S.Sarkar, ${ }^{39}$ J.Salicio, ${ }^{18}$ E.Sanchez, ${ }^{25}$ C.Schäfer ${ }^{18}$ V.Schegelsky, ${ }^{34}$ H.Schopper, ${ }^{21}$ D.J.Schotanus, ${ }^{31}$ C.Sciacca ${ }^{29}$ L.Servoli, ${ }^{33}$ S.Shevchenko, ${ }^{32}$ N.Shivarov ${ }^{42}$ V.Shoutko, ${ }^{13}$ E.Shumilov, ${ }^{28}$ A.Shvorob ${ }^{32}$ D.Son, ${ }^{43}$ C.Souga,${ }^{24}$ P.Spillantini ${ }^{17}$ M.Steuer, ${ }^{13}$ D.P.Stickland ${ }^{37}$ B.Stoyanov, ${ }^{42}$ A.Straessner, ${ }^{20}$ K.Sudhakar, G.Sultanov, ${ }^{42}$ L.Z.Sun, ${ }^{22}$ S.Sushkov, ${ }^{1}$ H.Suter, ${ }^{49}$ J.D.Swain, ${ }^{10}$ Z.Szillasi, ${ }^{26,}$ X.W.Tang, ${ }^{7}$ P.Tarjan, ${ }^{15}$ L.Tauscher, ${ }^{5}$ L.Taylor ${ }^{10}$ B.Tellili, ${ }^{24}$ D.Teyssier, ${ }^{24}$ C.Timmermans, ${ }^{31}$ Samuel C.C.Ting, ${ }^{13}$ S.M.Ting, ${ }^{13}$ S.C.Tonwar, J.Tóth, ${ }^{12}$ C.Tully, ${ }^{37}$ K.L.Tung, J.Ulbricht, ${ }^{49}$ E.Valente, ${ }^{39}$ R.T.Van de Walle, ${ }^{31}$ R.Vasquez, ${ }^{46}$ V.Veszpremi, ${ }^{26}$ G.Vesztergombi ${ }^{12}{ }^{2}$ I.Vetlitsky, ${ }^{28}$ D.Vicinanza, ${ }^{40}$ G.Viertel ${ }^{49}$ S.Villa, ${ }^{38}$ M.Vivargent, S.Vlachos, ${ }^{4}$ I.Vodopianov, ${ }^{26}$ H.Vogel ${ }^{35}$ H.Vogt ${ }^{48}$ I.Vorobiev, ${ }^{35,28}$ A.A.Vorobyov ${ }^{34}$ M.Wadhwa, Q.Wang ${ }^{31}$ X.L.Wang, ${ }^{22}$ Z.M.Wang, ${ }^{22}$ A.Weber, M.Weber, ${ }^{18}$ H.Wilkens, ${ }^{31}$ S.Wynhoff, ${ }^{37}$ L.Xia, ${ }^{32}$ Z.Z.Xu, ${ }^{22}$ J.Yamamoto, B.Z.Yang, ${ }^{22}$ C.G.Yang, H.J.Yang, M.Yang, S.C.Yeh, ${ }^{3}$ An.Zalite, ${ }^{34}$ Yu.Zalite, ${ }^{34}$ Z.P.Zhang, ${ }^{22}$ J.Zhao, ${ }^{22}$ G.Y.Zhu, R.Y.Zhu, ${ }^{32}$ H.L.Zhuang, A.Zichichi, ${ }^{8,18,19}$ B.Zimmermann, ${ }^{49}$ M.Zöller.' 
1 III. Physikalisches Institut, RWTH, D-52056 Aachen, Germany ${ }^{\S}$

2 National Institute for High Energy Physics, NIKHEF, and University of Amsterdam, NL-1009 DB Amsterdam, The Netherlands

3 University of Michigan, Ann Arbor, MI 48109, USA

4 Laboratoire d'Annecy-le-Vieux de Physique des Particules, LAPP,IN2P3-CNRS, BP 110, F-74941 Annecy-le-Vieux CEDEX, France

5 Institute of Physics, University of Basel, CH-4056 Basel, Switzerland

6 Louisiana State University, Baton Rouge, LA 70803, USA

7 Institute of High Energy Physics, IHEP, 100039 Beijing, China ${ }^{\triangle}$

8 University of Bologna and INFN-Sezione di Bologna, I-40126 Bologna, Italy

9 Tata Institute of Fundamental Research, Mumbai (Bombay) 400 005, India

10 Northeastern University, Boston, MA 02115, USA

11 Institute of Atomic Physics and University of Bucharest, R-76900 Bucharest, Romania

12 Central Research Institute for Physics of the Hungarian Academy of Sciences, H-1525 Budapest 114, Hungary ${ }^{\ddagger}$

13 Massachusetts Institute of Technology, Cambridge, MA 02139, USA

14 Panjab University, Chandigarh 160 014, India

15 KLTE-ATOMKI, H-4010 Debrecen, Hungary ${ }^{\circledR}$

16 Department of Experimental Physics, University College Dublin, Belfield, Dublin 4, Ireland

17 INFN Sezione di Firenze and University of Florence, I-50125 Florence, Italy

18 European Laboratory for Particle Physics, CERN, CH-1211 Geneva 23, Switzerland

19 World Laboratory, FBLJA Project, CH-1211 Geneva 23, Switzerland

20 University of Geneva, CH-1211 Geneva 4, Switzerland

21 University of Hamburg, D-22761 Hamburg, Germany

22 Chinese University of Science and Technology, USTC, Hefei, Anhui 230 029, China ${ }^{\triangle}$

23 University of Lausanne, CH-1015 Lausanne, Switzerland

24 Institut de Physique Nucléaire de Lyon, IN2P3-CNRS,Université Claude Bernard, F-69622 Villeurbanne, France

25 Centro de Investigaciones Energéticas, Medioambientales y Tecnológicas, CIEMAT, E-28040 Madrid, Spainb

26 Florida Institute of Technology, Melbourne, FL 32901, USA

27 INFN-Sezione di Milano, I-20133 Milan, Italy

28 Institute of Theoretical and Experimental Physics, ITEP, Moscow, Russia

29 INFN-Sezione di Napoli and University of Naples, I-80125 Naples, Italy

30 Department of Physics, University of Cyprus, Nicosia, Cyprus

31 University of Nijmegen and NIKHEF, NL-6525 ED Nijmegen, The Netherlands

32 California Institute of Technology, Pasadena, CA 91125, USA

33 INFN-Sezione di Perugia and Università Degli Studi di Perugia, I-06100 Perugia, Italy

34 Nuclear Physics Institute, St. Petersburg, Russia

35 Carnegie Mellon University, Pittsburgh, PA 15213, USA

36 INFN-Sezione di Napoli and University of Potenza, I-85100 Potenza, Italy

37 Princeton University, Princeton, NJ 08544, USA

38 University of Californa, Riverside, CA 92521, USA

39 INFN-Sezione di Roma and University of Rome, "La Sapienza", I-00185 Rome, Italy

40 University and INFN, Salerno, I-84100 Salerno, Italy

41 University of California, San Diego, CA 92093, USA

42 Bulgarian Academy of Sciences, Central Lab. of Mechatronics and Instrumentation, BU-1113 Sofia, Bulgaria

43 The Center for High Energy Physics, Kyungpook National University, 702-701 Taegu, Republic of Korea

44 National Central University, Chung-Li, Taiwan, China

45 Department of Physics, National Tsing Hua University, Taiwan, China

46 Purdue University, West Lafayette, IN 47907, USA

47 Paul Scherrer Institut, PSI, CH-5232 Villigen, Switzerland

48 DESY, D-15738 Zeuthen, Germany

49 Eidgenössische Technische Hochschule, ETH Zürich, CH-8093 Zürich, Switzerland

$\S$ Supported by the German Bundesministerium für Bildung, Wissenschaft, Forschung und Technologie.

¥ Supported by the Hungarian OTKA fund under contract numbers T019181, F023259 and T037350.

ๆ Also supported by the Hungarian OTKA fund under contract number T026178.

b Supported also by the Comisión Interministerial de Ciencia y Tecnología.

\# Also supported by CONICET and Universidad Nacional de La Plata, CC 67, 1900 La Plata, Argentina.

$\triangle$ Supported by the National Natural Science Foundation of China. 


\begin{tabular}{|c|cccccccc|}
\hline$\langle\sqrt{s}\rangle[\mathrm{GeV}]$ & 188.6 & 191.6 & 195.5 & 199.6 & 201.8 & 204.8 & 206.5 & 208.0 \\
\hline $\mathcal{L}\left[\mathrm{pb}^{-1}\right]$ & 176.8 & 29.8 & 84.1 & 83.3 & 37.1 & 79.0 & 130.5 & 8.6 \\
\hline
\end{tabular}

Table 1: Average centre-of-mass energies and integrated luminosities.

\begin{tabular}{|c|c|c|c|c|c|c|c|c|c|c|}
\hline \multirow[t]{2}{*}{ Selection } & \multicolumn{10}{|c|}{ Efficiencies [\%] for $\mathrm{e}^{+} \mathrm{e}^{-} \rightarrow$} \\
\hline & $\mathrm{e} \nu \mathrm{e} \nu$ & $\mathrm{e} \nu \mu \nu$ & $\mathrm{e} \nu \tau \nu$ & $\mu \nu \mu \nu$ & $\mu \nu \tau \nu$ & $\tau \nu \tau \nu$ & qqe $\nu$ & $\mathrm{qq} \mu \nu$ & $\mathrm{qq} \tau \nu$ & qqqq \\
\hline $\mathrm{e} \nu \mathrm{e} \nu$ & 54.7 & 0.8 & 11.4 & & 0.1 & 1.5 & & & & \\
\hline $\mathrm{e} \nu \mu \nu$ & & 47.6 & 8.4 & 1.4 & 10.1 & 2.2 & & & & \\
\hline $\mathrm{e} \nu \tau \nu$ & 6.0 & 1.7 & 27.8 & & 0.4 & 7.5 & & & & \\
\hline$\mu \nu \mu \nu$ & & & & 41.0 & 6.9 & 0.9 & & & & \\
\hline$\mu \nu \tau \nu$ & & 2.6 & 0.3 & 3.0 & 23.1 & 4.8 & & & & \\
\hline$\tau \nu \tau \nu$ & 0.2 & 0.1 & 2.1 & & 1.3 & 16.7 & & & & \\
\hline $\mathrm{qqe} \nu$ & & & & & & & 73.3 & 0.2 & 1.6 & \\
\hline $\mathrm{qq} \mu \nu$ & & & & & & & 0.1 & 74.2 & 4.2 & \\
\hline $\mathrm{qq} \tau \nu$ & & & & & & & 6.2 & 10.1 & 49.8 & 0.1 \\
\hline qqqq & & & & & & & 0.1 & & 0.4 & 84.0 \\
\hline
\end{tabular}

Table 2: Selection efficiencies for the signal processes $\mathrm{e}^{+} \mathrm{e}^{-} \rightarrow \ell \nu \ell \nu, \mathrm{e}^{+} \mathrm{e}^{-} \rightarrow \mathrm{qq} \ell \nu$, and $\mathrm{e}^{+} \mathrm{e}^{-} \rightarrow$ qqqq, at $\sqrt{s}=206.5 \mathrm{GeV}$. For the $\mathrm{e}^{+} \mathrm{e}^{-} \rightarrow$ qqqq selection, the numbers are quoted for a neural-network output greater than 0.6. Selection efficiencies at other centre-of-mass energies are only marginally different. 


\begin{tabular}{|c|c|c|c|c|c|c|c|c|}
\hline $\mathrm{e}^{+} \mathrm{e}^{-} \rightarrow$ & $N_{\text {data }}$ & $N_{\mathrm{bg}}$ & $\begin{array}{c}\sigma(\mathrm{CC} 03) \\
{[\mathrm{pb}]}\end{array}$ & $\begin{array}{l}\sigma_{\mathrm{SM}} \\
{[\mathrm{pb}]}\end{array}$ & $N_{\text {data }}$ & $N_{\mathrm{bg}}$ & $\begin{array}{c}\sigma(\mathrm{CC} 03) \\
{[\mathrm{pb}]}\end{array}$ & $\begin{array}{l}\sigma_{\mathrm{SM}} \\
{[\mathrm{pb}]}\end{array}$ \\
\hline & \multicolumn{4}{|c|}{$\langle\sqrt{s}\rangle=188.6 \mathrm{GeV}$} & \multicolumn{4}{|c|}{$\langle\sqrt{s}\rangle=201.8 \mathrm{GeV}$} \\
\hline$\ell \nu \ell \nu$ & 235 & 57.2 & $1.87 \pm 0.17 \pm 0.06$ & 1.72 & 40 & 12.3 & $1.47 \pm 0.35 \pm 0.07$ & 1.81 \\
\hline qqe $\nu$ & 347 & 22.9 & $2.29 \pm 0.14 \pm 0.03$ & 2.38 & 70 & 5.3 & $2.26 \pm 0.30 \pm 0.03$ & 2.49 \\
\hline $\mathrm{qq} \mu \nu$ & 341 & 14.9 & $2.25 \pm 0.14 \pm 0.04$ & 2.38 & 79 & 3.4 & $2.62 \pm 0.33 \pm 0.05$ & 2.49 \\
\hline $\mathrm{qq} \tau \nu$ & 413 & 69.7 & $2.82 \pm 0.22 \pm 0.07$ & 2.38 & 77 & 13.9 & $2.45 \pm 0.47 \pm 0.06$ & 2.49 \\
\hline \multirow[t]{2}{*}{ qqqq } & 1477 & 328.7 & $7.17 \pm 0.24 \pm 0.12$ & 7.42 & 301 & 64.6 & $7.10 \pm 0.52 \pm 0.12$ & 7.79 \\
\hline & \multicolumn{4}{|c|}{$\langle\sqrt{s}\rangle=191.6 \mathrm{GeV}$} & \multicolumn{4}{|c|}{$\langle\sqrt{s}\rangle=204.8 \mathrm{GeV}$} \\
\hline$\ell \nu \ell \nu$ & 35 & 10.4 & $1.67 \pm 0.41 \pm 0.07$ & 1.76 & 85 & 25.9 & $1.58 \pm 0.26 \pm 0.05$ & 1.82 \\
\hline qqe $\nu$ & 73 & 4.1 & $2.95 \pm 0.37 \pm 0.04$ & 2.42 & 176 & 11.0 & $2.78 \pm 0.23 \pm 0.04$ & 2.50 \\
\hline $\mathrm{qq} \mu \nu$ & 63 & 2.4 & $2.61 \pm 0.36 \pm 0.04$ & 2.42 & 142 & 6.5 & $2.30 \pm 0.22 \pm 0.04$ & 2.50 \\
\hline $\mathrm{qq} \tau \nu$ & 57 & 11.9 & $1.87 \pm 0.48 \pm 0.05$ & 2.42 & 164 & 26.4 & $2.63 \pm 0.33 \pm 0.07$ & 2.50 \\
\hline \multirow[t]{2}{*}{ qqqq } & 236 & 57.5 & $6.79 \pm 0.56 \pm 0.15$ & 7.56 & 656 & 137.2 & $7.66 \pm 0.37 \pm 0.13$ & 7.81 \\
\hline & \multicolumn{4}{|c|}{$\langle\sqrt{s}\rangle=195.5 \mathrm{GeV}$} & \multicolumn{4}{|c|}{$\langle\sqrt{s}\rangle=206.5 \mathrm{GeV}$} \\
\hline$\ell \nu \ell \nu$ & 105 & 30.2 & $1.76 \pm 0.25 \pm 0.06$ & 1.79 & 128 & 42.6 & $1.42 \pm 0.19 \pm 0.06$ & 1.82 \\
\hline $\mathrm{qqe} \nu$ & 168 & 10.9 & $2.36 \pm 0.20 \pm 0.03$ & 2.46 & 269 & 16.9 & $2.56 \pm 0.17 \pm 0.03$ & 2.50 \\
\hline $\mathrm{qq} \mu \nu$ & 157 & 8.2 & $2.14 \pm 0.20 \pm 0.03$ & 2.46 & 240 & 11.8 & $2.28 \pm 0.17 \pm 0.04$ & 2.50 \\
\hline $\mathrm{qq} \tau \nu$ & 222 & 33.8 & $3.44 \pm 0.34 \pm 0.08$ & 2.46 & 287 & 45.1 & $2.92 \pm 0.27 \pm 0.07$ & 2.50 \\
\hline \multirow[t]{2}{*}{ qqqq } & 665 & 153.5 & $6.92 \pm 0.34 \pm 0.11$ & 7.68 & 1108 & 220.1 & $8.07 \pm 0.29 \pm 0.13$ & 7.82 \\
\hline & \multicolumn{4}{|c|}{$\langle\sqrt{s}\rangle=199.6 \mathrm{GeV}$} & \multicolumn{4}{|c|}{$\langle\sqrt{s}\rangle=208.0 \mathrm{GeV}$} \\
\hline$\ell \nu \ell \nu$ & 87 & 26.0 & $1.68 \pm 0.27 \pm 0.06$ & 1.80 & 11 & 2.4 & $2.23 \pm 0.86 \pm 0.06$ & 1.82 \\
\hline $\mathrm{qqe} \nu$ & 152 & 11.4 & $2.21 \pm 0.20 \pm 0.03$ & 2.48 & 14 & 1.1 & $2.02 \pm 0.61 \pm 0.03$ & 2.50 \\
\hline $\mathrm{qq} \mu \nu$ & 142 & 7.3 & $2.05 \pm 0.20 \pm 0.04$ & 2.48 & 23 & 0.7 & $3.59 \pm 0.81 \pm 0.05$ & 2.50 \\
\hline $\mathrm{qq} \tau \nu$ & 181 & 32.2 & $2.75 \pm 0.32 \pm 0.07$ & 2.48 & 17 & 2.9 & $2.43 \pm 1.03 \pm 0.06$ & 2.50 \\
\hline qqqq & 726 & 151.1 & $7.91 \pm 0.36 \pm 0.13$ & 7.76 & 65 & 14.1 & $7.28 \pm 1.16 \pm 0.11$ & 7.82 \\
\hline
\end{tabular}

Table 3: Number of selected data events, $N_{\text {data }}$, number of expected background events, $N_{\text {bg }}$, not originating from $\mathrm{W}$-boson pair production, and CC03 cross sections for the reactions $\mathrm{e}^{+} \mathrm{e}^{-} \rightarrow$ $\ell \nu \ell \nu, \mathrm{e}^{+} \mathrm{e}^{-} \rightarrow \mathrm{qqe} \nu, \mathrm{e}^{+} \mathrm{e}^{-} \rightarrow \mathrm{qq} \mu \nu, \mathrm{e}^{+} \mathrm{e}^{-} \rightarrow \mathrm{qq} \tau \nu$, and $\mathrm{e}^{+} \mathrm{e}^{-} \rightarrow$ qqqq. For $\mathrm{e}^{+} \mathrm{e}^{-} \rightarrow \mathrm{qqqq}$, $N_{\text {data }}$ and $N_{\text {bg }}$ correspond to a cut on the output of the neural network at 0.6 , while the $\mathrm{e}^{+} \mathrm{e}^{-} \rightarrow \mathrm{qqqq}$ cross section is obtained from a fit to the neural-network output distribution, as described in Section 4. All cross sections are derived without any assumption on the W-boson decay branching fractions. The first uncertainty is statistical and the second systematic. Also shown are the Standard Model CC03 cross sections, $\sigma_{\mathrm{SM}}$, as calculated with YFSWW3 [16] with an uncertainty of $0.5 \%$. 


\begin{tabular}{|c|rc|}
\hline$\langle\sqrt{s}\rangle[\mathrm{GeV}]$ & \multicolumn{1}{|c|}{$\sigma_{\mathrm{qq}}^{\text {meas }}[\mathrm{pb}]$} & $\sigma_{\mathrm{qq}}^{\mathrm{MC}}[\mathrm{pb}]$ \\
\hline 188.6 & $107.5 \pm 3.4$ & 101.00 \\
191.6 & $92.8 \pm 7.6$ & 97.74 \\
195.5 & $86.7 \pm 4.5$ & 92.47 \\
199.6 & $86.8 \pm 4.7$ & 88.09 \\
201.8 & $89.6 \pm 7.0$ & 85.89 \\
204.8 & $84.1 \pm 4.7$ & 82.19 \\
206.5 & $78.1 \pm 3.6$ & 80.90 \\
\hline
\end{tabular}

Table 4: Measured, $\sigma_{\mathrm{qq}}^{\text {meas }}$, and expected, $\sigma_{\mathrm{qq}}^{\mathrm{MC}}$, cross sections of the $\mathrm{e}^{+} \mathrm{e}^{-} \rightarrow \mathrm{q} \overline{\mathrm{q}}(\gamma)$ process. The measurements are determined by a fit of the neural-network output distribution of the qqqq selection to both signal and $\mathrm{e}^{+} \mathrm{e}^{-} \rightarrow \mathrm{q} \overline{\mathrm{q}}(\gamma)$ background.

\begin{tabular}{|l||c|c|c|c|c|}
\hline \multicolumn{5}{|c|}{ Systematic uncertainties on $\sigma$ [\%] } \\
\hline \multirow{3}{*}{ Source } & \multicolumn{5}{|c|}{ Final state } \\
\cline { 2 - 6 } & $\ell \nu \ell \nu$ & qqe $\nu$ & qq $\mu \nu$ & qq $\tau \nu$ & qqqq \\
\hline Luminosity & \multicolumn{5}{|c|}{0.22} \\
MC statistics (signal) & 0.80 & 0.25 & 0.25 & 0.44 & 0.11 \\
MC statistics (background) & 1.57 & 0.23 & 0.28 & 0.75 & 0.22 \\
Detector modelling & 2.00 & 1.00 & 1.20 & 2.00 & 1.00 \\
Hadronisation (signal) & - & 0.77 & 0.58 & 1.17 & 0.45 \\
Hadronisation (background) & - & - & - & - & 0.90 \\
Bose-Einstein effects & - & \multicolumn{5}{|c|}{$<0.01$} & 0.03 \\
Colour reconnection & - & - & - & - & 0.19 \\
Background cross sections & 0.59 & 0.21 & 0.22 & 0.40 & 0.40 \\
W mass ( $\pm 0.04 \mathrm{GeV})$ & 0.27 & 0.03 & 0.03 & 0.10 & 0.06 \\
W width $( \pm 0.06 \mathrm{GeV})$ & 0.12 & 0.03 & 0.12 & 0.08 & 0.02 \\
\cline { 2 - 6 } ISR simulation & \multicolumn{5}{|c|}{$<0.01$} \\
\cline { 2 - 6 } FSR simulation & 0.21 & 0.21 & 0.17 & 0.08 & $<0.01$ \\
\hline Total & 2.76 & 1.36 & 1.43 & 2.52 & 1.46 \\
\hline
\end{tabular}

Table 5: Relative systematic uncertainties on the cross-section measurements evaluated for $\sqrt{s}=206.5 \mathrm{GeV}$. Uncertainties at other center-of-mass energies are only marginally different. 


\begin{tabular}{|c||c|c|c||c|}
\hline$\sigma[\mathrm{pb}]$ & $\langle\sqrt{s}\rangle=188.6 \mathrm{GeV}$ & $\langle\sqrt{s}\rangle=191.6 \mathrm{GeV}$ & $\langle\sqrt{s}\rangle=195.5 \mathrm{GeV}$ & $\langle\sqrt{s}\rangle=199.6 \mathrm{GeV}$ \\
\hline$\sigma_{\ell \nu \ell \nu}$ & $1.88 \pm 0.16 \pm 0.07$ & $1.66 \pm 0.39 \pm 0.07$ & $1.78 \pm 0.24 \pm 0.07$ & $1.75 \pm 0.25 \pm 0.06$ \\
$\sigma_{\mathrm{qq} \ell \nu}$ & $7.19 \pm 0.24 \pm 0.08$ & $7.69 \pm 0.61 \pm 0.09$ & $7.58 \pm 0.36 \pm 0.08$ & $6.81 \pm 0.35 \pm 0.08$ \\
$\sigma_{\mathrm{qqqq}}$ & $7.17 \pm 0.24 \pm 0.12$ & $6.78 \pm 0.56 \pm 0.12$ & $6.92 \pm 0.34 \pm 0.11$ & $7.91 \pm 0.36 \pm 0.13$ \\
\hline$\sigma_{\mathrm{WW}}$ & $16.17 \pm 0.37 \pm 0.17$ & $16.11 \pm 0.89 \pm 0.17$ & $16.22 \pm 0.54 \pm 0.16$ & $16.49 \pm 0.55 \pm 0.17$ \\
\hline$\sigma_{\mathrm{SM}}$ & 16.27 & 16.57 & 16.84 & 17.02 \\
\hline \hline$\sigma[\mathrm{pb}]$ & $\langle\sqrt{s}\rangle=201.8 \mathrm{GeV}$ & $\langle\sqrt{s}\rangle=204.8 \mathrm{GeV}$ & $\langle\sqrt{s}\rangle=206.5 \mathrm{GeV}$ & $\langle\sqrt{s}\rangle=208.0 \mathrm{GeV}$ \\
\hline$\sigma_{\ell \nu \ell \nu}$ & $1.51 \pm 0.34 \pm 0.07$ & $1.58 \pm 0.24 \pm 0.05$ & $1.44 \pm 0.18 \pm 0.06$ & $2.23 \pm 0.86 \pm 0.06$ \\
$\sigma_{\mathrm{qq} \ell \nu}$ & $7.34 \pm 0.54 \pm 0.08$ & $7.68 \pm 0.39 \pm 0.13$ & $7.60 \pm 0.30 \pm 0.08$ & $8.18 \pm 1.21 \pm 0.09$ \\
$\sigma_{\mathrm{qqqq}}$ & $7.09 \pm 0.52 \pm 0.12$ & $7.66 \pm 0.37 \pm 0.13$ & $8.07 \pm 0.29 \pm 0.13$ & $7.29 \pm 1.16 \pm 0.11$ \\
\hline$\sigma_{\mathrm{WW}}$ & $16.01 \pm 0.81 \pm 0.17$ & $17.00 \pm 0.58 \pm 0.17$ & $17.31 \pm 0.45 \pm 0.18$ & $17.52 \pm 1.81 \pm 0.17$ \\
\hline$\sigma_{\mathrm{SM}}$ & 17.08 & 17.12 & 17.14 & 17.15 \\
\hline
\end{tabular}

Table 6: Measured CC03 cross sections of the processes $\mathrm{e}^{+} \mathrm{e}^{-} \rightarrow \ell \nu \ell \nu, \mathrm{e}^{+} \mathrm{e}^{-} \rightarrow \mathrm{qq} \ell \nu$ (summed over lepton flavours) and $\mathrm{e}^{+} \mathrm{e}^{-} \rightarrow$ qqqq, assuming charged-lepton universality. The measured W-boson pair-production cross sections, $\sigma_{\mathrm{Ww}}$, are derived assuming Standard Model branching fractions for the $\mathrm{W}$ boson decay modes. The Standard Model total W-boson pair-production cross sections, $\sigma_{\mathrm{SM}}$, are calculated using the YFSWW3 program, which has a theoretical uncertainty of $0.5 \%$.

\begin{tabular}{|c||c|c||c|}
\hline $\begin{array}{c}\text { Branching } \\
\text { fraction }\end{array}$ & $\begin{array}{c}\text { Lepton } \\
\text { non-universality }\end{array}$ & $\begin{array}{c}\text { Lepton } \\
\text { universality }\end{array}$ & $\begin{array}{c}\text { Standard } \\
\text { Model }\end{array}$ \\
\hline \hline $\mathrm{Br}(\mathrm{W} \rightarrow \mathrm{e} \nu)[\%]$ & $10.78 \pm 0.29 \pm 0.13$ & - & \\
$\mathrm{Br}(\mathrm{W} \rightarrow \mu \nu)[\%]$ & $10.03 \pm 0.29 \pm 0.12$ & - & \\
$\mathrm{Br}(\mathrm{W} \rightarrow \tau \nu)[\%]$ & $11.89 \pm 0.40 \pm 0.20$ & - & 10.83 \\
$\mathrm{Br}(\mathrm{W} \rightarrow \ell \nu)[\%]$ & - & $10.83 \pm 0.14 \pm 0.10$ & 67.51 \\
$\mathrm{Br}(\mathrm{W} \rightarrow \mathrm{qq})[\%]$ & $67.30 \pm 0.42 \pm 0.30$ & $67.50 \pm 0.42 \pm 0.30$ & \\
\hline
\end{tabular}

Table 7: W-boson decay branching fractions derived without and with the assumption of charged-lepton universality. The correlation coefficients between the leptonic branching fractions are $-0.016,-0.279,-0.295$ for $[\operatorname{Br}(\mathrm{W} \rightarrow \mathrm{e} \nu), \operatorname{Br}(\mathrm{W} \rightarrow \mu \nu)],[\operatorname{Br}(\mathrm{W} \rightarrow \mathrm{e} \nu), \operatorname{Br}(\mathrm{W} \rightarrow \tau \nu)]$ and $[\operatorname{Br}(\mathrm{W} \rightarrow \mu \nu), \operatorname{Br}(\mathrm{W} \rightarrow \tau \nu)]$, respectively. The W-boson decay branching fractions expected in the Standard Model are also listed. 


\begin{tabular}{|c||c|c||c|c|}
\cline { 2 - 5 } \multicolumn{1}{c||}{} & \multicolumn{4}{c|}{$\mathrm{d} \sigma / \mathrm{d} \cos \theta_{\mathrm{W}^{-}}[\mathrm{pb}]$} \\
\hline $\cos \theta_{\mathrm{W}}$ - range & $\langle\sqrt{s}\rangle=182.7$ & $\mathrm{SM}$ & $\langle\sqrt{s}\rangle=189.0$ & $\mathrm{SM}$ \\
\hline$-1.0,-0.8$ & $0.54 \pm 0.23 \pm 0.01$ & 0.74 & $0.69 \pm 0.12 \pm 0.01$ & 0.64 \\
$-0.8,-0.6$ & $0.81 \pm 0.29 \pm 0.01$ & 0.84 & $0.88 \pm 0.15 \pm 0.01$ & 0.78 \\
$-0.6,-0.4$ & $0.22 \pm 0.26 \pm 0.00$ & 1.02 & $1.08 \pm 0.17 \pm 0.02$ & 0.94 \\
$-0.4,-0.2$ & $0.96 \pm 0.33 \pm 0.01$ & 1.20 & $1.18 \pm 0.19 \pm 0.02$ & 1.14 \\
$-0.2, \quad 0.0$ & $1.71 \pm 0.43 \pm 0.03$ & 1.44 & $1.34 \pm 0.20 \pm 0.02$ & 1.38 \\
$0.0, \quad 0.2$ & $2.27 \pm 0.50 \pm 0.03$ & 1.78 & $1.51 \pm 0.22 \pm 0.02$ & 1.72 \\
$0.2, \quad 0.4$ & $3.37 \pm 0.62 \pm 0.05$ & 2.16 & $1.88 \pm 0.24 \pm 0.03$ & 2.22 \\
$0.4, \quad 0.6$ & $3.52 \pm 0.66 \pm 0.05$ & 2.86 & $2.95 \pm 0.31 \pm 0.04$ & 2.95 \\
$0.6, \quad 0.8$ & $4.24 \pm 0.74 \pm 0.06$ & 3.84 & $4.19 \pm 0.37 \pm 0.06$ & 4.15 \\
$0.8, \quad 1.0$ & $5.00 \pm 0.83 \pm 0.07$ & 5.47 & $6.11 \pm 0.47 \pm 0.09$ & 6.24 \\
\hline
\end{tabular}

\begin{tabular}{|c||c|c||c|c|}
\cline { 2 - 5 } \multicolumn{1}{c||}{} & \multicolumn{4}{c|}{$\mathrm{d} \sigma / \mathrm{d} \cos \theta_{\mathrm{W}^{-}}[\mathrm{pb}]$} \\
\hline $\cos \theta_{\mathrm{W}^{-}}$range & $\langle\sqrt{s}\rangle=198.3$ & $\mathrm{SM}$ & $\langle\sqrt{s}\rangle=205.9$ & $\mathrm{SM}$ \\
\hline$-1.0,-0.8$ & $0.68 \pm 0.11 \pm 0.01$ & 0.57 & $0.60 \pm 0.10 \pm 0.01$ & 0.52 \\
$-0.8,-0.6$ & $0.76 \pm 0.13 \pm 0.01$ & 0.71 & $0.44 \pm 0.11 \pm 0.01$ & 0.64 \\
$-0.6,-0.4$ & $0.78 \pm 0.15 \pm 0.01$ & 0.85 & $0.77 \pm 0.14 \pm 0.01$ & 0.78 \\
$-0.4,-0.2$ & $0.80 \pm 0.16 \pm 0.01$ & 1.05 & $0.99 \pm 0.16 \pm 0.01$ & 0.98 \\
$-0.2, \quad 0.0$ & $1.31 \pm 0.20 \pm 0.02$ & 1.29 & $1.35 \pm 0.20 \pm 0.02$ & 1.21 \\
$0.0, \quad 0.2$ & $1.64 \pm 0.23 \pm 0.02$ & 1.65 & $1.72 \pm 0.23 \pm 0.03$ & 1.55 \\
$0.2, \quad 0.4$ & $2.21 \pm 0.27 \pm 0.03$ & 2.16 & $1.75 \pm 0.23 \pm 0.03$ & 2.06 \\
$0.4, \quad 0.6$ & $2.41 \pm 0.29 \pm 0.04$ & 2.97 & $2.84 \pm 0.30 \pm 0.04$ & 2.92 \\
$0.6, \quad 0.8$ & $3.69 \pm 0.36 \pm 0.05$ & 4.38 & $4.80 \pm 0.41 \pm 0.07$ & 4.45 \\
$0.8, \quad 1.0$ & $6.26 \pm 0.49 \pm 0.09$ & 7.20 & $7.49 \pm 0.53 \pm 0.11$ & 7.80 \\
\hline
\end{tabular}

Table 8: Sum of the differential cross sections, as function of $\cos \theta_{\mathrm{W}^{-}}$, for the $\mathrm{e}^{+} \mathrm{e}^{-} \rightarrow \mathrm{qqe} \nu$ and $\mathrm{e}^{+} \mathrm{e}^{-} \rightarrow \mathrm{qq} \mu \nu$ processes. The measurements are derived in a restricted phase space of the CC03 subset of diagrams. The first uncertainty is statistical and the second is systematic. The systematic uncertainty is fully correlated between $\cos \theta_{\mathrm{W}^{-}}$bins and between $\sqrt{s}$ bins. The columns labeled SM show the expected values from the Standard Model, which have a theoretical uncertainty of about $2 \%$. 

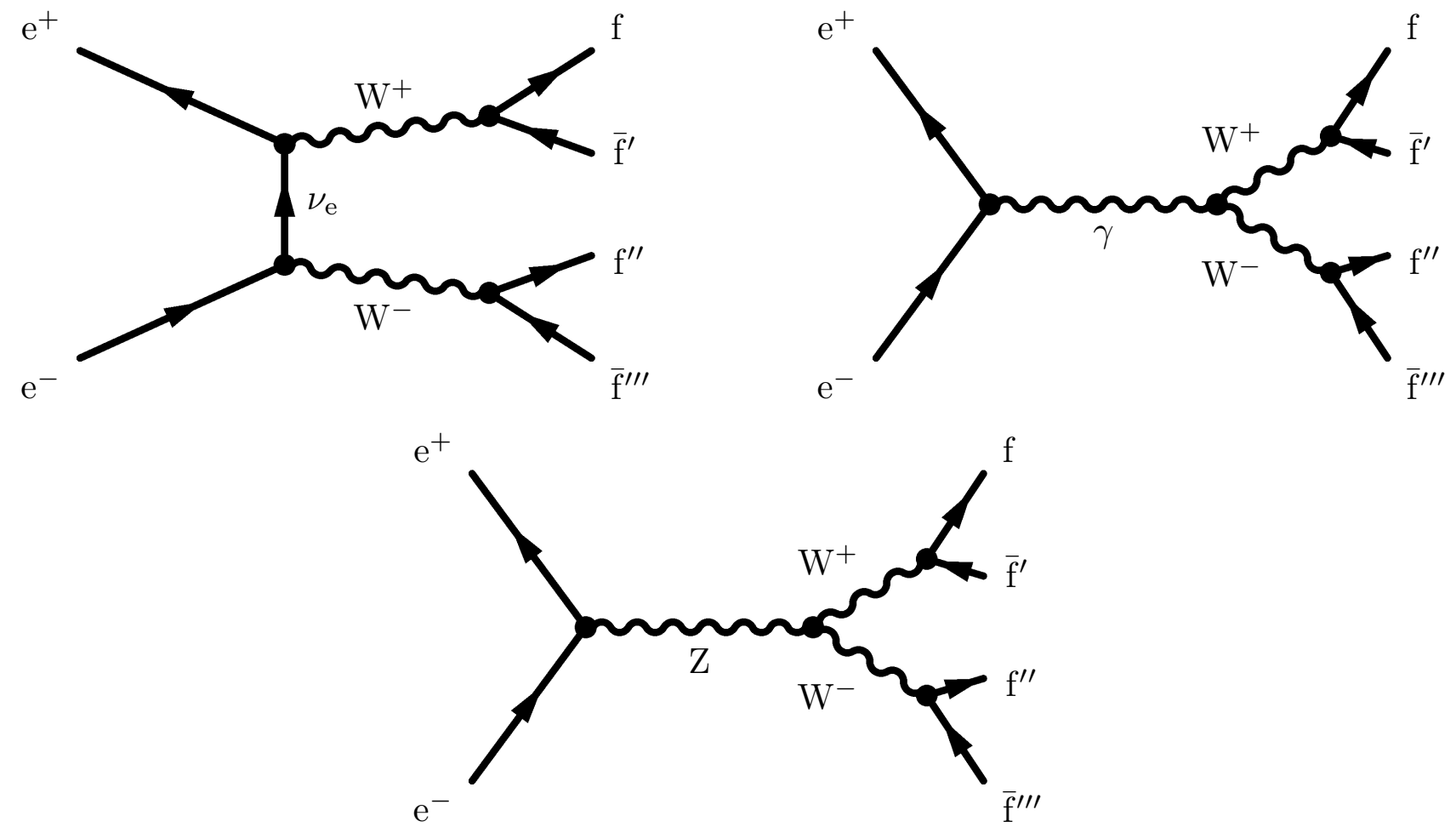

Figure 1: The lowest-order Feynman diagrams (CC03) contributing to W-boson pair production: $t$-channel $\nu$ exchange and $s$-channel $\gamma$ and Z-boson exchange. 


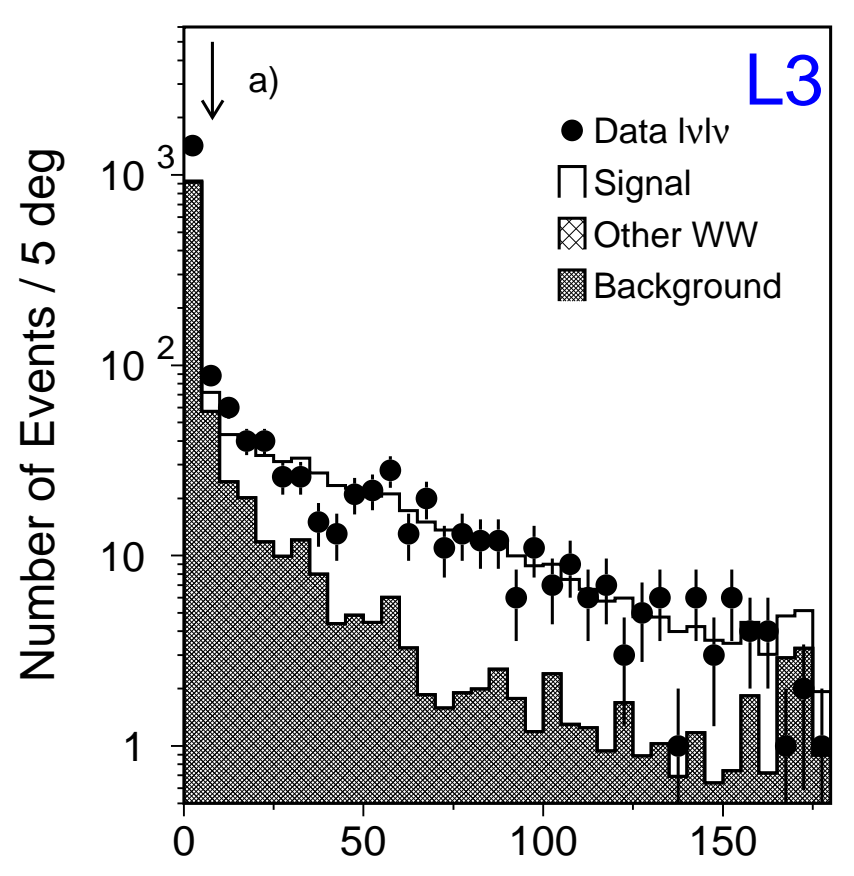

Lepton-Lepton Acoplanarity (deg)

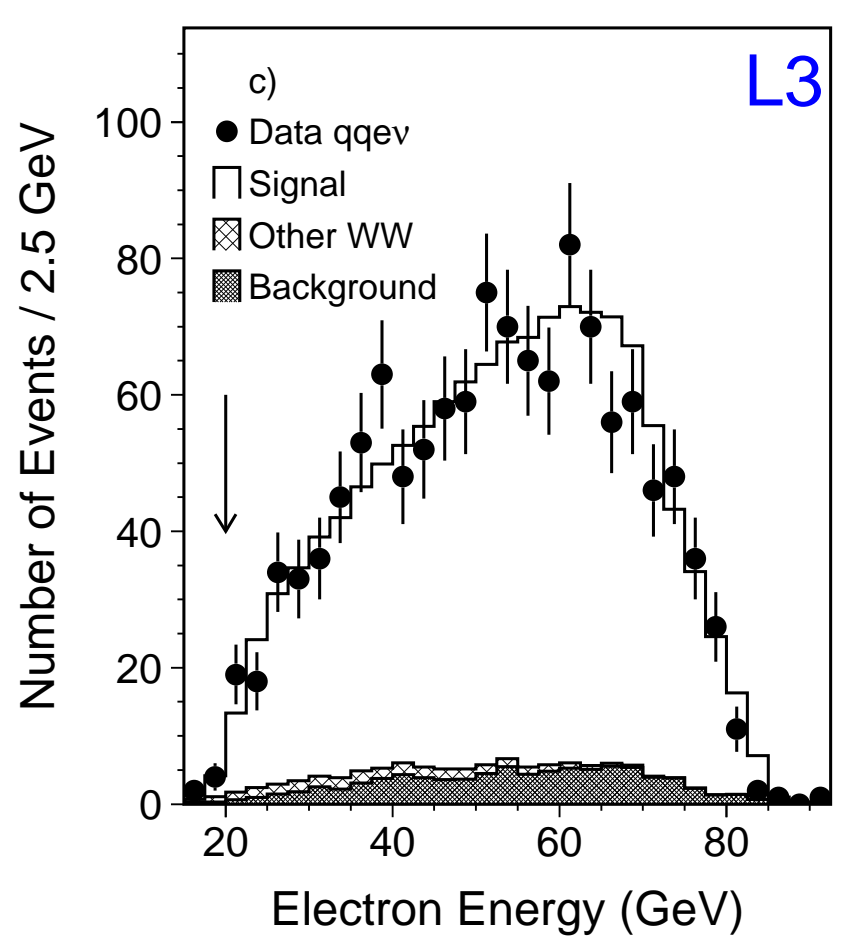

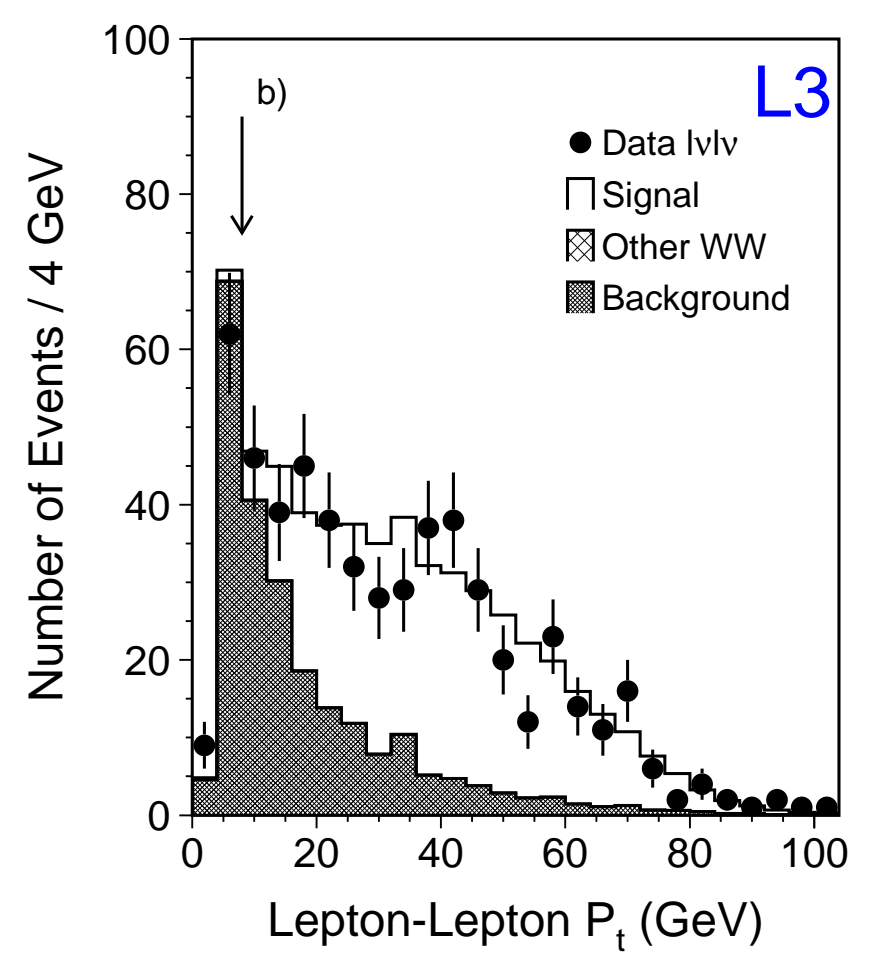

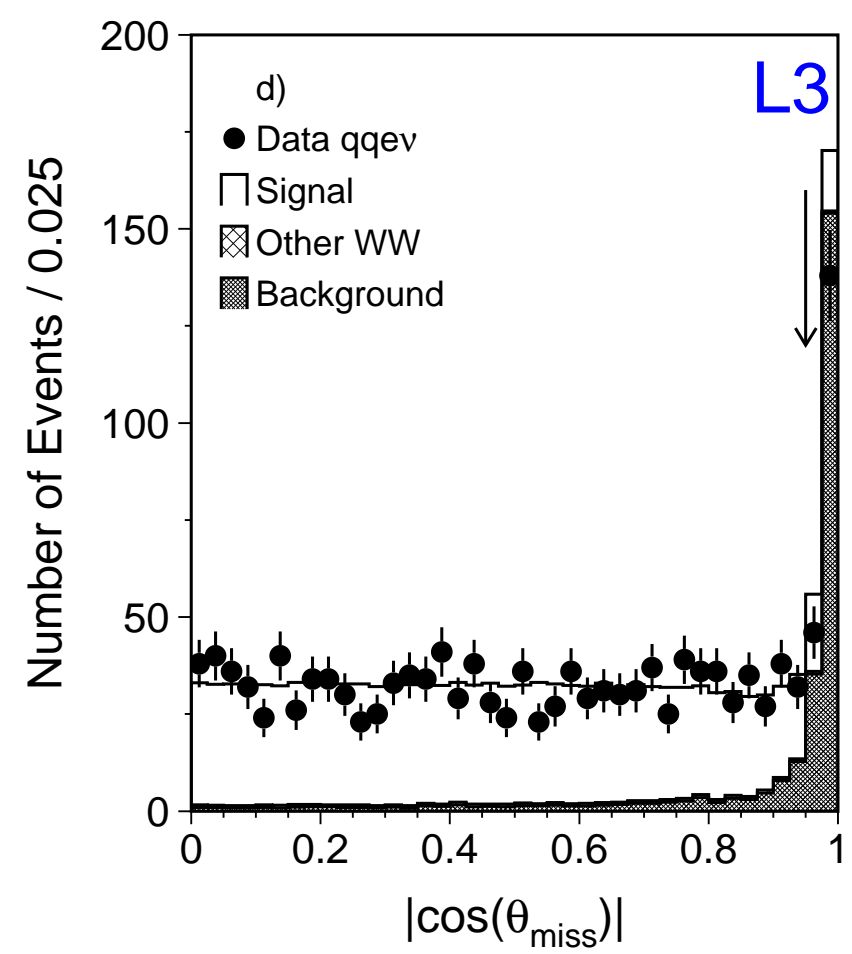

Figure 2: Distributions of variables used for the selection of $\ell \nu \ell \nu$ and qqe $\nu$ events, comparing the signal and background Monte Carlo to the data collected at $\sqrt{s}=189-209 \mathrm{GeV}$. The positions of the selection cuts are indicated by vertical arrows. All selection cuts except the one on the plotted variable are applied. (a) The acoplanarity between the two leptons in the lepton-lepton class of the $\ell \nu \ell \nu$ selection. The excess in the first bin is due to cosmic-ray background. (b) The momentum transverse to the beam direction of the selected $\ell \nu \ell \nu$ events in the lepton-lepton class. (c) The energy of the electron in qqe $\nu$ events. (d) The absolute value of the cosine of the polar angle of the missing momentum in qqe $\nu$ events. 

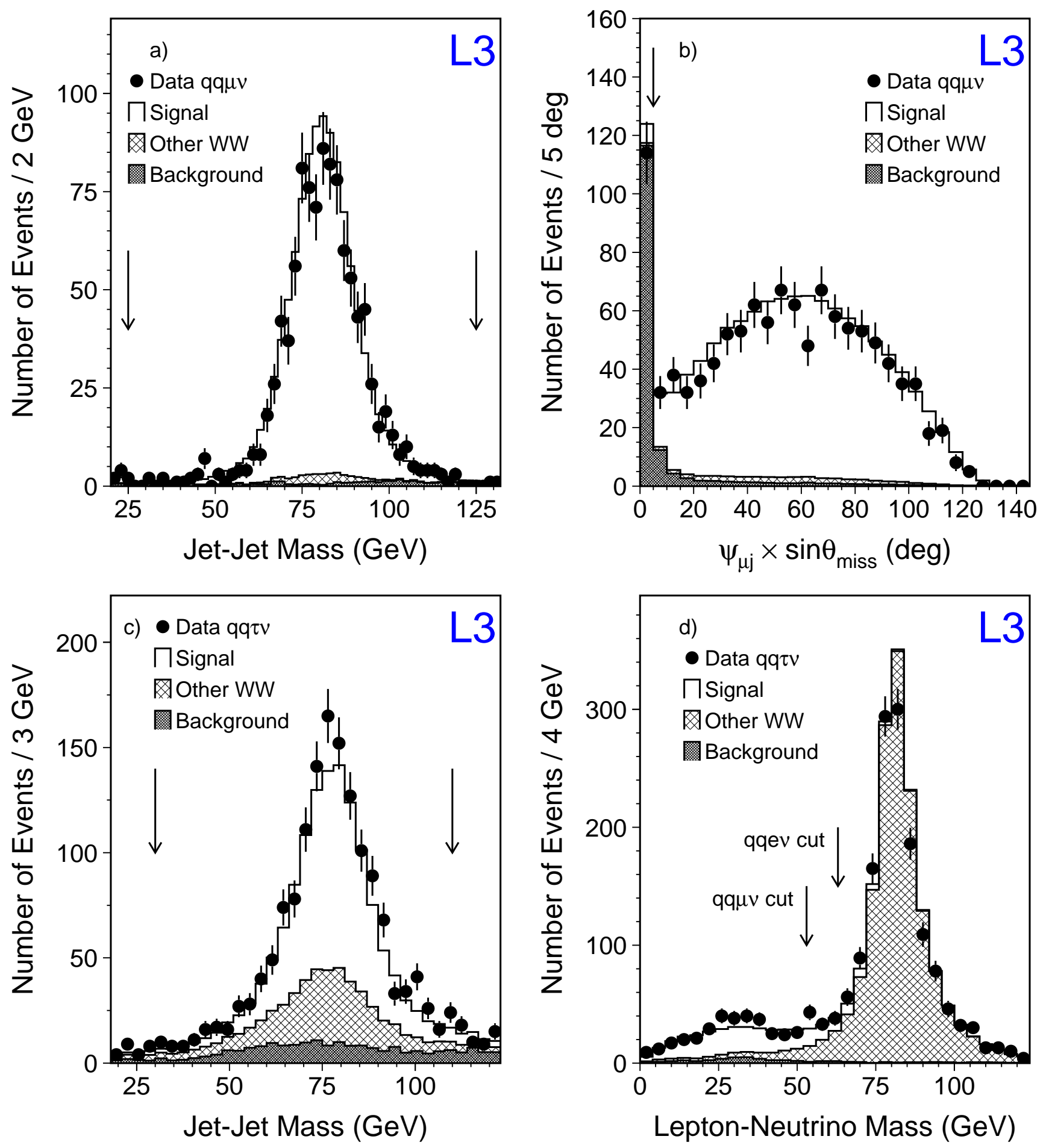

Figure 3: Distributions of variables used for the selection of qq $\mu \nu$ and qq $\tau \nu$ events, comparing the signal and background Monte Carlo to the data collected at $\sqrt{s}=189-209 \mathrm{GeV}$. (a) The invariant mass of the jet-jet system in qq $\mu \nu$ events. (b) The quantity $\psi_{\mu j} \times \sin \theta_{\text {miss }}$ in qq $\mu \nu$ events. (c) The invariant mass of the jet-jet system in qq $\tau \nu$ events. (d) The invariant mass of the lepton-neutrino system for leptonically decaying tau candidates in qq $\tau \nu$ events. 

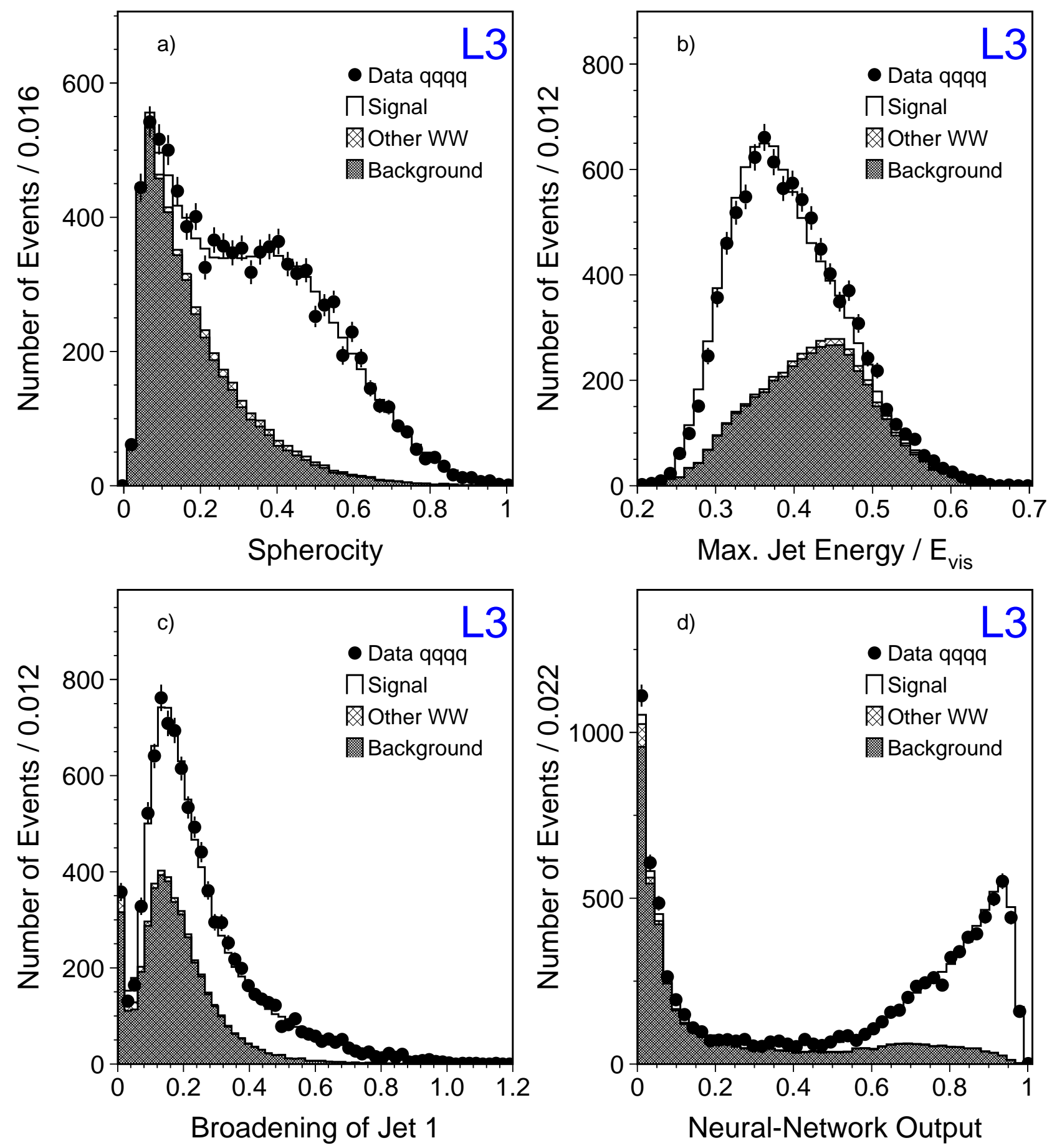

Figure 4: Distributions of some of the variables used for the neural network in the analysis of qqqq events together with the neural-network output, comparing the signal and background Monte Carlo to the data collected at $\sqrt{s}=189-209 \mathrm{GeV}$. (a) The spherocity. (b) The maximum jet energy scaled by the visible energy. (c) The broadening of the most energetic jet. (d) The neural-network output. 


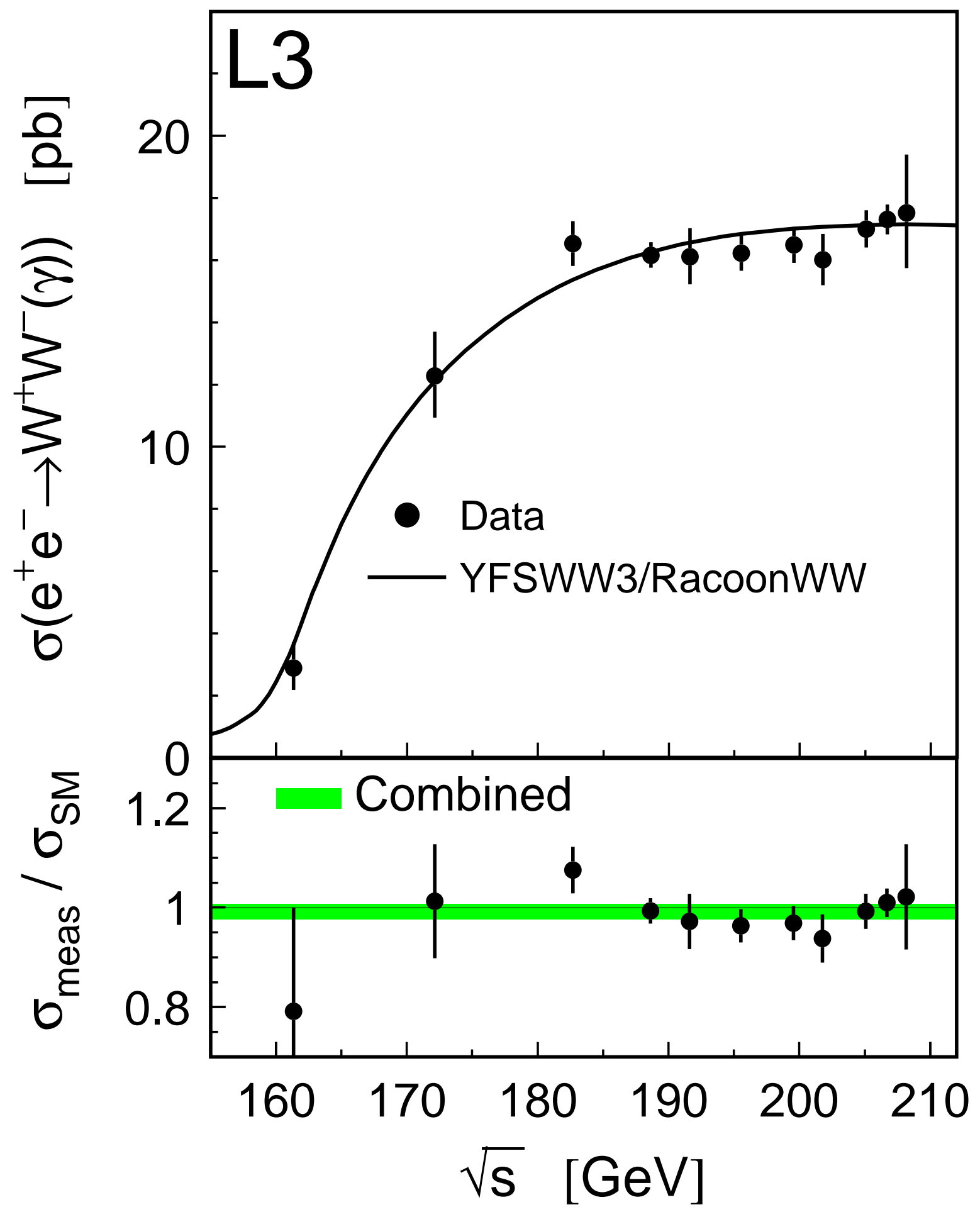

Figure 5: The cross section of the process $\mathrm{e}^{+} \mathrm{e}^{-} \rightarrow \mathrm{W}^{+} \mathrm{W}^{-}$as a function of $\sqrt{s}$. The published measurements of $\sigma_{\mathrm{WW}}$ at $\sqrt{s}$ of $161 \mathrm{GeV}, 172 \mathrm{GeV}$ and $183 \mathrm{GeV}$, the updated measurement at $\sqrt{s}=189 \mathrm{GeV}$ and the new measurements at $\sqrt{s}=192-209 \mathrm{GeV}$ are shown as dots with error bars, combining statistical and systematic uncertainties in quadrature. The solid curve shows the Standard Model expectation as calculated with YFSWW3 in the whole energy range and RacoonWW for $\sqrt{s} \geq 170 \mathrm{GeV}$. Its uncertainty of $0.5 \%$ is invisible on this scale. The lower plot shows the ratios of the measured cross sections with respect to the Standard Model expectations as calculated with YFSWW3. The band represent their combined value with its total uncertainty: $R=0.992 \pm 0.015$. 

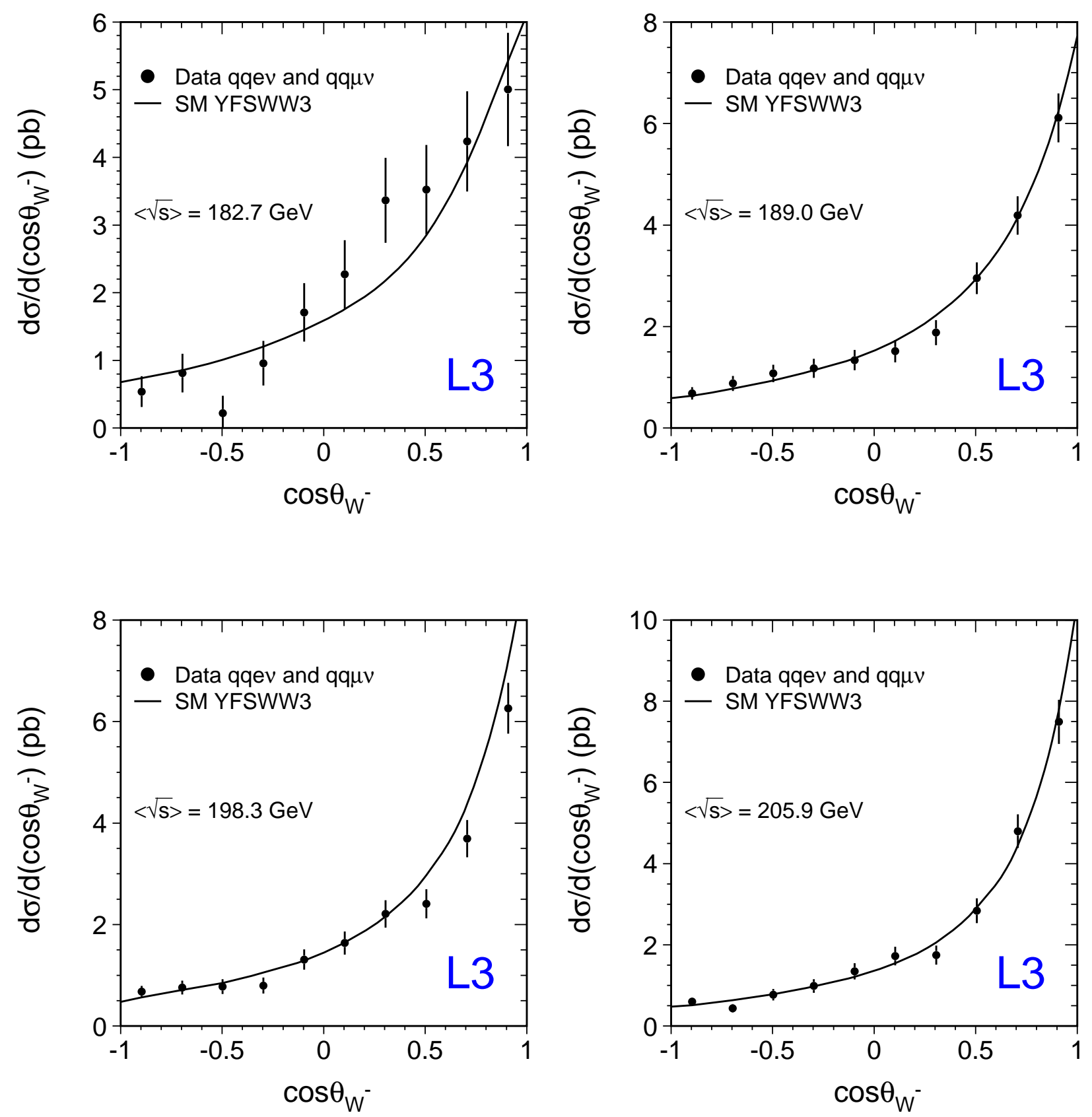

Figure 6: Measured differential cross sections as a function of $\cos \theta_{\mathrm{W}^{-}}$for the $\mathrm{e}^{+} \mathrm{e}^{-} \rightarrow \mathrm{qqe} \nu$ and $\mathrm{e}^{+} \mathrm{e}^{-} \rightarrow \mathrm{qq} \mu \nu$ processes. The cross sections of the two channels are summed. Experimental data are represented by dots with error bars which include statistical and systematic uncertainties added in quadrature. Monte Carlo expectations are shown as solid lines. 